\title{
THE ROLE OF SAMPLING FOR STABILITY AND PERFORMANCE IN UNCONSTRAINED NONLINEAR MODEL PREDICTIVE CONTROL*
}

\author{
KARL WORTHMANN ${ }^{\dagger}$, MARCUS REBLE ${ }^{\ddagger}$, LARS GRÜNE§ ${ }^{\S}$ AND FRANK ALLGÖWER
}

\begin{abstract}
We investigate the impact of sampling on stability and performance estimates in nonlinear model predictive control without stabilizing terminal constraints. Interpreting the sampling period as a discretization parameter, the relation between continuous and discrete time estimates depending on this parameter is analyzed. The technique presented in this paper allows to determine the sampling rate required in order to approximate the continuous time suboptimality bound arbitrarily well and, thus, gives insight into the trade-off between sampling time and guaranteed performance.
\end{abstract}

Key words. nonlinear model predictive control, performance guarantee, suboptimality estimate, sampling rate, multistep feedback laws, discretization, receding horizon control, relaxed Lyapunov inequality.

AMS subject classifications. 93C10, 93C20, 93C57, 93B05, 93D15, 93D20, 93D05

1. Introduction. Model predictive control (MPC), often also termed receding horizon control (RHC), is a successful modern control method due to its ability to take performance criteria directly into account and to guarantee satisfaction of hard constraints [23]. However, it is well known that if the prediction horizon is chosen too short, MPC can destabilize even open-loop stable linear systems. A practical (nonlinear) example was shown in [22], for which MPC with a short finite prediction horizon yields an unstable closed-loop.

Stability of MPC for nonlinear systems has been extensively studied over the last decades. Most MPC schemes with guaranteed asymptotic stability make use of additional terminal costs and/or constraints, cf. $[18,6]$. For more details, we refer to $[2,23]$ and the references therein.

An alternative are so called unconstrained $M P C$ formulations, which have received significant attention in recent years. Note that the notion unconstrained refers here to the absence of stabilizing terminal constraints in the MPC formulation, but input and state constraints can be taken into account in these control schemes. These unconstrained MPC schemes are attractive because the computation time needed for the online optimization may be reduced and more suitable numerical methods are available when no terminal constraint is imposed. Several approaches, such as $[12,16,7]$, use control Lyapunov functions as terminal weighting functions and remove the terminal constraint from the finite horizon optimal control problem. Due to additional conditions, the terminal constraints are implicitly guaranteed to be satisfied. The additional requirement of control Lyapunov functions as terminal weighting functions was removed in $[13,8,9]$. These unconstrained MPC schemes are of interest in particular due to three reasons: First, the absence of terminal constraints facilitates the derivation of performance bounds on the infinite horizon performance of the MPC closed-loop. Second, the unconstrained MPC formulation does not require the

* This work was supported by the DFG priority research program 1305: Control Theory of Digitally Networked Dynamical Systems.

$\dagger$ University of Bayreuth, 95440 Bayreuth, Germany, (karl.worthmann@uni-bayreuth.de).

$\ddagger$ University of Stuttgart, 70550 Stuttgart, Germany, (marcus.reble@ist.uni-stuttgart.de).

$\S$ University of Bayreuth, 95440 Bayreuth, Germany, (lars.gruene@uni-bayreuth.de).

ฯUniversity of Stuttgart, 70550 Stuttgart, Germany, (frank.allgower@ist.uni-stuttgart.de). 
knowledge of a local control Lyapunov function. Third, unconstrained MPC without terminal weighting is widely used in industrial applications [21].

However, the stability analysis of these unconstrained MPC formulations requires different techniques compared to classical stability results for MPC with terminal costs and/or constraints, cf. $[14,5]$. It was shown in [13] that if the prediction horizon is chosen sufficiently large, stability of the closed-loop can be guaranteed. However, no explicit bounds on stabilizing prediction horizons were given. The first explicit bound on a stabilizing prediction horizon was deduced in [8] and later improved in [28]. The main ingredient in the stability analysis was the knowledge of an upper bound of the optimal cost function. Independently, a typically smaller bound on the minimal stabilizing prediction horizon was given in $[9,10]$ using a related controllability assumption, but a significantly distinct analysis. The results use the solution of a linear program, whose construction is based on the assumed controllability condition and Bellman's principle of optimality, in order to guarantee a decrease of the MPC cost functional along the corresponding closed-loop trajectory. For a comparison between both approaches we refer to [29, Section 5.5]. The results from $[9,10]$ have been extended to continuous time systems in $[24,25]$. In the continuous time case, the linear program has infinitely many decision variables and constraints - but a solution can be derived due to its particular structure.

In the present work we are concerned with the connection of the discrete and the continuous time approaches. A comparison of the continuous time methodology with the sampled-data implementation of its discrete time counterpart in [25] indicated a gap between the respective performance estimates - despite the similarity of both techniques. In this comparison, the sampling period can be considered as a discretization parameter. The main contribution of the present paper is to close this gap. The key ingredient to achieve this goal is to decouple the sampling period and the length of the interval on which the MPC control function is applied. To this end, the concept of multistep feedback laws introduced in [9] is employed. This multistep feedback gives a natural framework to take more information about the continuous time system into account and, as a consequence, allows to recover the continuous time horizon estimate as a limiting case and thus closes the observed gap. Furthermore, we show that the continuous time results represent an upper bound in terms of guaranteed performance for the discrete time results applied in a sampled-data context. Indeed, the technique to be presented allows to determine the required sampling rate in order to approximate this performance estimate arbitrarily well and, thus, gives insight into the trade-off between sampling time and performance.

The remainder of this work is organized as follows. In Section 2, we present the problem setup to be considered and recall previous results on unconstrained MPC. Section 2.1 is concerned with a continuous time setting whereas Section 2.2 discusses unconstrained MPC for discrete time systems. In addition, a discretization is introduced in order to connect both settings. The main results of this paper are presented in Section 3. We show that the performance estimates obtained from the discrete time setting are monotonically increasing for so called iterative refinements and converge, for a discretization parameter tending to zero, to an upper bound which can be computed in the continuous time setting. In Section 4 our results are illustrated for an example of a reaction diffusion equation. Conclusions are drawn in Section 5.

Notation: Let $\mathbb{N}$ and $\mathbb{R}$ denote the natural and the real numbers, respectively. In addition, the notation $\mathbb{N}_{0}=\mathbb{N} \cup\{0\}$ is used. $\mathbb{R}^{n}$ denotes the $n$-dimensional Euclidean space with an arbitrary norm $\|\cdot\| \cdot\lfloor s\rfloor$ denotes the largest integer smaller or equal to 
$s$ and $\lceil s\rceil$ denotes the smallest integer larger or equal to $s$. Furthermore, a continuous function $\eta: \mathbb{R}_{\geq 0} \rightarrow \mathbb{R}_{\geq 0}$ is said to belong to class $\mathcal{K}_{\infty}$ if it is strictly increasing, unbounded, and satisfies $\eta(0)=0$.

For the example of a partial differential equation considered in Subsection 4.1, the following definitions of function spaces are required:

$$
\begin{aligned}
\mathcal{L}^{\infty}(\Omega) & :=\{z: \Omega \rightarrow \mathbb{R} \mid z(\cdot) \text { is measurable and essentially bounded }\}, \\
\mathcal{L}^{2}(\Omega) & :=\left\{z: \Omega \rightarrow \mathbb{R} \mid z(\cdot) \text { is measurable and }\|z(\cdot)\|_{\mathcal{L}^{2}(\Omega)}=\sqrt{\int_{\Omega} z(x)^{2} d x}<\infty\right\}, \\
\mathcal{H}^{1}(\Omega) & :=\left\{z: \Omega \rightarrow \mathbb{R} \mid z(\cdot) \in \mathcal{L}^{2}(\Omega) \text { and } D_{i} z(\cdot) \in \mathcal{L}^{2}(\Omega)\right\} .
\end{aligned}
$$

Here, $\Omega \subset \mathbb{R}^{n}$ denotes an open domain and $D_{i} z(\cdot)$ the weak derivative with respect to the $i$ th component of the independent space variable $x$. Furthermore, a function $z: \Omega \rightarrow \mathbb{R}$ is essentially bounded if there exists a constant $M \in \mathbb{R}$ such that $|z(x)|<M$ holds almost everywhere. $\mathcal{C}(\Omega)$ represents the space of continuous functions on $\Omega$ and a subscript, e.g., $\mathcal{C}_{0}(\Omega)$, indicates that the support of the functions contained in the respective class of functions is compact. In addition, the space

$$
W(0, T):=\left\{z: z \in \mathcal{L}^{2}\left([0, T), \mathcal{H}^{1}(\Omega)\right) \text { and } z_{t} \in \mathcal{L}^{2}\left([0, T), \mathcal{H}^{1}(\Omega)^{*}\right)\right\}
$$

is required. $\mathcal{C}([0, T), B)$ represents the class of continuous abstract functions on $[0, T)$ mapping into a normed Banach space $B$. An abstract $\mathcal{L}^{2}$-function is measurable and square integrable in the sense of Bochner, cf. [27] for details. $\mathcal{H}^{1}(\Omega)^{*}$ denotes the dual of $\mathcal{H}^{1}(\Omega)$.

2. Unconstrained Model Predictive Control. We consider nonlinear continuous time control systems

$$
\dot{z}(t)=f(z(t), u(t))
$$

with state space $Z$ and set $U$ of control values. Here, $Z$ and $U$ are allowed to be arbitrary metric spaces in order to deal with ordinary and partial differential equations. $z_{u}\left(\cdot ; z_{0}\right)=z\left(\cdot ; z_{0}, u(\cdot)\right)$ denotes the solution of the controlled differential equation (2.1) which is manipulated by the function $u(\cdot): \mathbb{R}_{\geq 0} \rightarrow U$ and emanates from the initial state $z_{u}\left(0 ; z_{0}\right)=z_{0} \in Z$. The subsets $\mathbb{Z} \subseteq Z$ and $\mathbb{U} \subseteq U$ denote the set of feasible states and controls, respectively. If the conditions

$$
u(t) \in \mathbb{U}, t \in[0, T) \quad \text { and } \quad z_{u}\left(t ; z_{0}\right) \in \mathbb{Z}, t \in[0, T]
$$

hold, $u(\cdot)$ is called admissible on $[0, T) . \mathcal{U}[0, T)$ denotes the set of all admissible control functions on $[0, T)$ and $\mathcal{U}[0, \infty)$ represents the set of control functions $u(\cdot)$ satisfying $u(\cdot) \in \mathcal{U}[0, T)$ for each $T>0$. We point out that the set $\mathcal{U}[0, T), T \in \mathbb{R}_{>0} \cup\{\infty\}$, of admissible control functions depends on $z_{0}$. Our main objective is to steer the system to an equilibrium $z^{\star} \in \mathbb{Z}$ which satisfies $f\left(z^{\star}, u^{\star}\right)=0$ for some $u^{\star} \in \mathbb{U}$.

2.1. Unconstrained MPC for Continuous Time Systems. In order to design a performance criterion which suitably characterizes the equilibrium $z^{\star}$, continuous stage costs $\ell: Z \times U \rightarrow \mathbb{R}_{\geq 0}$ are defined such that the conditions

$$
\ell\left(z^{\star}, u^{\star}\right)=0 \quad \text { and } \quad \ell(z, u) \geq \eta_{1}\left(d_{Z}\left(z, z^{\star}\right)\right),(z, u) \in \mathbb{Z} \times \mathbb{U},
$$


hold with a $\mathcal{K}_{\infty}$-function $\eta_{1}: \mathbb{R}_{\geq 0} \rightarrow \mathbb{R}_{\geq 0}$. Here, $d_{Z}: Z \times Z \rightarrow \mathbb{R}_{\geq 0}$ denotes the metric on $Z$. Based on these stage costs, a cost functional as well as the corresponding optimal value function are defined by

$$
J_{T}\left(z_{0}, u(\cdot)\right)=\int_{0}^{T} \ell\left(z_{u}\left(t ; z_{0}\right), u(t)\right) d t \quad \text { and } \quad V_{T}\left(z_{0}\right):=\inf _{u(\cdot) \in \mathcal{U}[0, T)} J_{T}\left(z_{0}, u(\cdot)\right)
$$

with prediction horizon $T \in \mathbb{R}_{>0} \cup\{\infty\}$.

Assumption 2.1. We assume that $V_{\infty}\left(z_{0}\right)<\infty$ holds for each $z_{0} \in \mathbb{Z}$. In addition, we suppose that, for given $T \in \mathbb{R}_{>0}$, the infimum in (2.2) is attained in order to avoid technical difficulties.

Ideally we would like to minimize the cost functional $J_{\infty}\left(z_{0}, \cdot\right)$. However, since infinite horizon optimal control problems are, in general, computationally intractable, we employ model predictive control in order to approximately solve this task. Firstly, set $\hat{z}:=z_{0}$. Then, MPC is composed of three steps:

(1) Solve the optimal control problem on a truncated and, thus, finite time horizon $T \in \mathbb{R}_{>0}$, i.e., find a control function $u^{\star}(\cdot) \in \mathcal{U}[0, T)$ satisfying

$$
J_{T}\left(\hat{z}, u^{\star}(\cdot)\right)=V_{T}(\hat{z}) .
$$

(2) For a constant control horizon $\delta \in(0, T)$, define the feedback law $\mu_{T, \delta}$ : $[0, \delta) \times Z \rightarrow U$ by $\mu_{T, \delta}(t, \hat{z})=u^{\star}(t), t \in[0, \delta)$, i.e., the MPC feedback equals the first portion of the computed control function $u^{\star}(\cdot)$. Then, implement $\left.\mu_{T, \delta}(t, \hat{z})\right|_{t \in[0, \delta)}$ at the plant. Doing so yields

$$
z_{\mu_{T, \delta}}(\delta ; \hat{z})=z\left(\delta ; \hat{z}, \mu_{T, \delta}(\cdot, \hat{z})\right) .
$$

(3) Shift the prediction (optimization) horizon forward in time, i.e., initialize the optimal control problem with $\hat{z}=z_{\mu_{T, \delta}}(\delta ; \hat{z})$ and repeat from step (1).

Supposing feasibility, iterative application of this methodology provides a control on the infinite time horizon. The resulting closed loop control and its corresponding solution are denoted by $\mu_{T, \delta}^{M P C}\left(\cdot ; z_{0}\right)$ and $z_{\mu_{T, \delta}}^{M P C}\left(\cdot ; z_{0}\right)$, respectively.

Our main tool in order to ensure stability of the resulting closed loop is the relaxed Lyapunov inequality

$$
V_{T}\left(z_{\mu_{T, \delta}}(\delta ; \hat{z})\right) \leq V_{T}(\hat{z})-\alpha \int_{0}^{\delta} \ell\left(z_{\mu_{T, \delta}}(t ; \hat{z}), \mu_{T, \delta}(t, \hat{z})\right) d t
$$

with $\alpha \in(0,1]$, cf. $[17,11]$. This inequality has to be shown for each state $\hat{z}$ of the MPC closed-loop trajectory $z_{\mu_{T, \delta}}^{M P C}\left(n \delta ; z_{0}\right), n \in \mathbb{N}_{0}$. Combining (2.3) with

$$
\eta_{1}\left(d_{Z}\left(z, z^{\star}\right)\right) \leq \min _{u \in \mathbb{U}} \ell(z, u)=: \ell^{\star}(z) \quad \text { and } \quad V_{T}(z) \leq \eta_{2}\left(d_{Z}\left(z, z^{\star}\right)\right)
$$

for all $z \in \mathbb{Z}$, in which $\eta_{1}(\cdot)$ and $\eta_{2}(\cdot)$ are class $\mathcal{K}_{\infty}$-functions, ensures asymptotic stability of the MPC closed loop, cf. [24, Proposition 1]. Hence, our goal is to establish the relaxed Lyapunov Inequality (2.3). To this end, we pursue an approach introduced in $[25,24]$ which is based on the following assumption.

Assumption 2.2. Let $C \geq 1$ and $\mu>0$ be given. Suppose that, for each $z_{0} \in \mathbb{Z}$, a control function $u_{z_{0}}(\cdot) \in \mathcal{U}[0, \infty)$ exists which satisfies

$$
\ell\left(z_{u_{z_{0}}}\left(t ; z_{0}\right), u_{z_{0}}(t)\right) \leq C e^{-\mu t} \ell^{\star}\left(z_{0}\right) \quad \text { for all } t \in \mathbb{R}_{\geq 0} .
$$


Assumption 2.2 is an exponential controllability assumption in terms of the stage cost $\ell(\cdot, \cdot)$ with overshoot constant $C$ and decay rate $\mu$. We recapitulate the main result deduced in [24]. Note that this theorem holds for our infinite dimensional setting and with slight modifications also for settings including delay equations, cf. $[26]$.

Theorem 2.3. Suppose that Assumption 2.2 and Inequality (2.4) hold. Furthermore, let $T>\delta>0$ and $\bar{\alpha} \in(0,1)$ be chosen such that

$$
\alpha_{T, \delta}:=1-\frac{\left(e^{\mu \delta}-1\right)^{1 / C}}{\left(e^{\mu T}-1\right)^{1 / C}-\left(e^{\mu \delta}-1\right)^{1 / C}} \cdot \frac{\left(e^{\mu(T-\delta)}-1\right)^{1 / C}}{\left(e^{\mu T}-1\right)^{1 / C}-\left(e^{\mu(T-\delta)}-1\right)^{1 / C}} \geq \bar{\alpha}
$$

holds. Then, for each $z_{0} \in \mathbb{Z}$, the MPC closed loop solution $z_{\mu_{T, \delta}}^{M P C}\left(\cdot ; z_{0}\right)$ is asymptotically stable and satisfies the suboptimality bound

$$
J_{\infty}\left(z_{0}, \mu_{T, \delta}^{M P C}\left(\cdot ; z_{0}\right)\right)=\int_{0}^{\infty} \ell\left(z_{\mu_{T, \delta}}^{M P C}\left(t ; z_{0}\right), \mu_{T, \delta}^{M P C}\left(t ; z_{0}\right)\right) d t \leq \frac{V_{\infty}\left(z_{0}\right)}{\bar{\alpha}} .
$$

Inequality (2.7) gives a performance estimates which compares the resulting MPC closed loop costs with the theoretically achievable minimal costs on the infinite time horizon. Here, the inherent monotonicity of unconstrained MPC schemes is crucial in order to deduce this bound on the, in general, unknown quantity $V_{\infty}(\cdot)$. Note that, for given suboptimality index $\bar{\alpha} \in(0,1)$, Condition (2.6) always holds for a suitably chosen prediction horizon $T$, cf. [24, Section 4.1].

2.2. Unconstrained MPC for Discretized Continuous Time Systems. Typically, continuous time controllers are implemented in a sampled-data fashion. To this end, a discretization parameter $\tau$, which is also called sampling period, is required. The corresponding time axis $\tau \mathbb{N}_{0}$ consists of the time instants $0, \tau, 2 \tau, 3 \tau, \ldots$. Furthermore, $W_{\tau}:=\{\tilde{u}:[0, \tau) \rightarrow U\}$ denotes the set of control values, i.e., a discrete time control value corresponds to a function $\tilde{u}(\cdot)$ defined on the (sampling) interval $[0, \tau)$. Then, the discrete time system dynamics are defined by

$$
z(n+1)=f_{\tau}(z(n), w(n))=\Phi(\tau ; z(n), w(n)(\cdot)), \quad z(0)=z_{0} .
$$

Here $\Phi(\tau ; z(n), w(n)(\cdot))$ denotes the solution of the differential equation (2.1) emanating from the initial value $z(n)$ and governed by the control function $w(n)(\cdot)$ at time $\tau$. Analogously to Subsection 2.1 existence and uniqueness of $\Phi(\cdot ; z(n), w(n))$ are tacitly assumed. The control constraints are incorporated by choosing $\mathbb{W}:=\{\tilde{u}:[0, \delta) \rightarrow \mathbb{U}\}$. For $N \in \mathbb{N}$ and $z_{0} \in \mathbb{Z}$, a sequence of control values $w(n), n=0,1,2, \ldots, N-1$, is called admissible if the conditions

$$
w(n) \in \mathbb{W} \quad \text { and } \quad \Phi(t ; z(n), w(n)(\cdot)) \in \mathbb{Z} \quad \forall t \in[0, \delta]
$$

are satisfied for all $n \in\{0,1,2, \ldots, N-1\}$ with $z(0)=z_{0} . \mathcal{W}_{N}=\mathcal{W}_{N}\left(z_{0}\right)$ represents the set of all admissible sequences of length $N$. Furthermore, $\mathcal{W}_{\infty}=\mathcal{W}_{\infty}\left(z_{0}\right)$ contains all sequences $(w(n))_{n \in \mathbb{N}_{0}}$ whose restriction to its first $N$ elements is contained in $\mathcal{W}_{N}$ for all $N \in \mathbb{N}$.

Next, we want to adapt the presented MPC algorithm to our discrete time setting. To this end, the corresponding prediction and control horizon are defined by $N:=T / \tau$ and $m:=\delta / \tau$, respectively. In addition, discrete time stage costs $\ell_{\tau}: Z \times W \rightarrow \mathbb{R}_{\geq 0}$ 
satisfying $\ell_{\tau}\left(z^{\star}, w^{\star}\right)=0$ for $w^{\star}(t)=u^{\star}, t \in[0, \delta)$, and $\ell_{\tau}^{\star}(z):=\min _{w \in \mathcal{W}_{1}(z)} \ell_{\tau}(z, w) \geq$ $\widetilde{\eta}_{1}\left(d_{Z}\left(z, z^{\star}\right)\right)$ for some $\widetilde{\eta}_{1} \in \mathcal{K}_{\infty}$ have to be defined. We point out that the discrete time running costs can reproduce the continuous time costs $\ell(\cdot, \cdot)$ on the corresponding sampling interval exactly but may also be designed according to some other criterion. Here we allow for completely independent functions for which, however, we will assume similar inequalities to hold. These preliminary considerations enable us to define the cost functional

$$
J_{N}\left(z_{0}, w(\cdot)\right)=\sum_{n=0}^{N-1} \ell_{\tau}\left(z_{w}\left(n ; z_{0}\right), w(n)\right)
$$

for $N \in \mathbb{N} \cup \infty$. Here, $z_{w}\left(n ; z_{0}\right)=z\left(n ; z_{0}, w(\cdot)\right)$ denotes the solution emanating from $z_{0}$ with sequence of control values $w(n), n=0,1,2, \ldots, N-1$. The optimal value function is defined analogously to the continuous time setting, i.e., $V_{N}\left(z_{0}\right):=$ $\inf _{w(\cdot) \in \mathcal{W}_{N}} J_{N}\left(z_{0}, w(\cdot)\right)$. Again, an MPC feedback is defined. However, since we aim at comparing results from the discrete time with the continuous time setting, we need to decouple the sampling interval from the control horizon, which is achieved by the following definition of a multistep feedback law.

Definition 2.4. Let $N \in \mathbb{N}_{\geq 2}$ and $m \in\{1,2, \ldots, N-1\}$ be given. A multistep feedback law is a map $\mu_{N, m}: Z \times\{0,1, \ldots, m-1\} \rightarrow W$ which is applied according to the rule $z_{\mu_{N, m}}(0 ; \hat{z})=\hat{z}$,

$$
z_{\mu_{N, m}}(n+1 ; \hat{z})=f_{\tau}\left(z_{\mu_{N, m}}(n ; \hat{z}), \mu_{N, m}(\hat{z}, n)\right), \quad n=0,1, \ldots, m-1 .
$$

Let $w^{\star}(\cdot) \in \mathcal{W}_{N}(\hat{z})$ be a sequence of control values minimizing $J_{N}(\hat{z}, w(\cdot))$. Then, the MPC multistep feedback $\mu_{N, m}(\cdot, \cdot)$ is defined by $\mu_{N, m}(\hat{z}, n)=w_{N}^{\star}(n)$ for $n=$ $0,1, \ldots, m-1$.

The MPC closed loop control and solution generated by the multistep feedback law $\mu_{N, m}$ are denoted by $\mu_{N, m}^{M P C}\left(\cdot ; z_{0}\right)$ and $z_{\mu_{N, m}}^{M P C}\left(\cdot ; z_{0}\right)$, respectively. For $m=1$ the feedback law from Definition 2.4 equals "classical" MPC, i.e, only the first control move is applied. The feedback law coincides with applying the first $m$ values of the sequence of control values which minimizes the cost functional $J_{N}(\hat{z}, \cdot)$ for the current state $\hat{z}$. Then, the prediction horizon is shifted and the next MPC iteration is carried out.

In order to conclude asymptotic stability of (2.8), a discrete time counterpart to our exponential controllability Assumption 2.2 is needed.

Assumption 2.5. Let $C \geq 1$ and $\sigma \in(0,1)$ be given. Then, for each $z_{0} \in \mathbb{Z}$, there exists $\left(w_{z_{0}}(n)\right)_{n \in \mathbb{N}_{0}} \in \mathcal{W}_{\infty}$ such that

$$
\ell_{\tau}\left(z_{w_{z_{0}}}(n), w_{z_{0}}(n)\right) \leq C \sigma^{n} \min _{w \in \mathcal{W}_{1}} \ell_{\tau}\left(z_{0}, w\right)=C \sigma^{n} \ell_{\tau}^{\star}\left(z_{0}\right) .
$$

Based on this assumption we formulate our main stability theorem, cf. $[9,10]$.

Theorem 2.6. Let $N \in \mathbb{N}_{\geq 2}, m \in\{1,2, \ldots, N-1\}$, and $\bar{\alpha} \in(0,1)$ be given as well as Assumption 2.5 be satisfied with $C \geq 1$ and $\sigma \in(0,1)$. Furthermore, assume that $\mathcal{K}_{\infty}$-functions $\widetilde{\alpha}_{1}(\cdot), \widetilde{\alpha}_{2}(\cdot)$ exist satisfying

$$
\widetilde{\alpha}_{1}\left(d_{Z}\left(z, z^{\star}\right)\right) \leq \ell_{\tau}^{\star}(z) \leq \widetilde{\alpha}_{2}\left(d_{Z}\left(z, z^{\star}\right)\right) \quad \forall z \in \mathbb{Z} .
$$


In addition, let the inequality

$$
\alpha_{N, m}:=1-\frac{\prod_{i=m+1}^{N}\left(\gamma_{i}-1\right) \prod_{i=N-m+1}^{N}\left(\gamma_{i}-1\right)}{\left(\prod_{i=m+1}^{N} \gamma_{i}-\prod_{i=m+1}^{N}\left(\gamma_{i}-1\right)\right)\left(\prod_{i=N-m+1}^{N} \gamma_{i}-\prod_{i=N-m+1}^{N}\left(\gamma_{i}-1\right)\right)} \geq \bar{\alpha}
$$

hold with $\gamma_{i}:=C \sum_{n=0}^{i-1} \sigma^{i}=C\left(1-\sigma^{i}\right) /(1-\sigma)$. Then, for each $z_{0} \in \mathbb{Z}, z_{\mu_{N, m}}^{M P C}\left(\cdot ; z_{0}\right)$ is asymptotically stable and the following suboptimality estimate holds

$$
J_{\infty}\left(z_{0}, \mu_{N, m}^{M P C}\left(\cdot ; z_{0}\right)\right)=\sum_{n=0}^{\infty} \ell_{\tau}\left(z_{\mu_{N, m}}^{M P C}\left(n ; z_{0}\right), \mu_{N, m}^{M P C}\left(n ; z_{0}\right)\right) \leq \frac{V_{\infty}\left(z_{0}\right)}{\bar{\alpha}},
$$

i.e., the cost assoziated with the MPC closed loop on $[0, \infty)$ are bounded by the optimal costs multiplied by $1 / \bar{\alpha}$.

REMARK 2.7 (Sampled-data systems with zero order hold). We point out that if Assumption 2.5 holds for control functions which are constant on each sampling interval $[n \tau,(n+1) \tau), n \in \mathbb{N}_{0} \cap[0, T / \tau)$, then we can conclude asymptotic stability of the corresponding sampled-data system with zero order hold. This conclusion cannot be drawn for the continuous time setting because the proof of Theorem 2.3 requires that switching between different control signals is possible at each time instant.

3. Connections between the Continuous and the Discrete Time Results. Although the discrete and the continuous time setups use similar controllability assumptions, a gap between the corresponding suboptimality Estimates (2.7) and (2.11) was observed in [25]. To be more precise, the continuous time analysis yielded significantly tighter performance bounds than the discrete time analysis and, thus, allowed to conclude asymptotic stability for smaller prediction horizons. This behavior was due to the fact that the control horizon was chosen equal to the length of the sampling interval. Since Theorem 2.6 is based on multistep feedback laws, this coupling can be avoided in order to overcome this gap. In particular, by investigating the impact of the discretization parameter $\tau$ on the suboptimality index $\alpha$ from Theorem 2.6, we show that the continuous time estimate can be approximated arbitrarily well by choosing the discretization parameter $\tau$ sufficiently small.

Let the prediction horizon $T$ and the parameter $\delta$, which determines the portion of the computed control function to be implemented, be fixed. Furthermore, suppose that Assumption 2.5 holds for each discretization parameter smaller than a given $\tau_{0} \leq \delta$. The following definition provides the needed technical tool in order to tackle the asked questions.

Definition 3.1 (Discretization and Iterative Refinement). Let $\tau_{0} \in(0, \delta]$ be chosen such that the conditions $m:=\delta / \tau_{0} \in \mathbb{N}$ and $N:=T / \tau_{0} \in \mathbb{N}$ hold. In addition, let a decay rate $\sigma_{0}=\sigma$ be given and assume that the overshoot $C$ does not depend on the discretization parameter $\tau$. Furthermore, let a sequence $\left(k_{j}\right)_{j \in \mathbb{N}_{0}} \subseteq \mathbb{N}$ with $k_{0}:=1$ be given. Then, a discretization $\mathcal{D}$ is defined by a sequence of quintruplets

$$
\mathcal{D}=\left(\mathcal{D}_{j}\right)_{j \in \mathbb{N}_{0}}=\left(k_{j}, \tau_{j}, N_{j}, m_{j}, \sigma_{j}\right)_{j \in \mathbb{N}_{0}}:=\left(k_{j}, k_{j}^{-1} \tau_{0}, k_{j} N, k_{j} m, \sigma^{k_{j}^{-1}}\right)_{j \in \mathbb{N}_{0}} .
$$

Here, $\left(\tau_{j}\right)_{j \in \mathbb{N}_{0}}, \tau_{j}:=\tau_{0} / k_{j}$, represents the sequence of discretization parameters and the parameters $\left(N_{j}\right)_{j \in \mathbb{N}_{0}}$ and $\left(m_{j}\right)_{j \in \mathbb{N}_{0}}$ specify the MPC feedback laws $\left(\mu_{N_{j}, m_{j}}\right)_{j \in \mathbb{N}_{0}}$. Note that also the decay rates of Assumption 2.5 are adapted depending on $\tau$. If $k_{j+1}$ 
is a multiple of $k_{j}$ and $k_{j+1}>k_{j}$ holds, a sequence $\left(k_{j}\right)_{j \in \mathbb{N}_{0}} \subset \mathbb{N}$ is called an iterative refinement.

In the $j$-th discretization the interval $\left[0, \tau_{0}\right)$ is subdivided into $k_{j}$ equidistant parts, i.e., $\left[0, \tau_{0} / k_{j}\right),\left[\tau_{0} / k_{j}, 2 \tau_{0} / k_{j}\right), \ldots,\left[\left(k_{j}-1\right) \tau_{0} / k_{j}, \tau_{0}\right)$. The discrete time dynamics $\left(f_{\tau_{j}}\right)_{j \in \mathbb{N}_{0}}$ as well as the stage costs $\left(\ell_{\tau_{j}}\right)_{j \in \mathbb{N}_{0}}$ are also adapted depending on the parameter $\tau_{j}$.

The multistep feedback law from Definition 2.4 is employed in order to compare the discretized and the continuous time setting. In the following the overshoot constants from Assumptions 2.2 and 2.5 are assumed to be equal. Additionally, suppose that $\sigma=\sigma_{0}=e^{-\mu \tau_{0}}$ holds. We comment on these assumptions in Subsection 4.2.

Our goal is to show that Theorem 2.3, which deals with the continuous time setting, can be interpreted as a limit of the discrete time results given in Theorem 2.6 for discretizations whose discretization parameters $\tau_{j}$ tend to zero for $j$ approaching infinity. Hence, the continuous time MPC feedback law $\mu_{T, \delta}$ is approximated by its discrete time counterpart $\mu_{k_{j} N, k_{j} m}$.

For the derivation of the expression $\alpha_{T, \delta}$ in Theorem 2.3, the controllability assumption and, in particular, Condition (2.5) is taken into account for all $t \in \mathbb{R}_{\geq 0}$. In contrast to that, the discrete time results rely only on Inequality (2.9) at the sampling instants, but no further information about times in between sampling instants is taken into account. Loosely speaking, less information about the actual system is used in the discrete time setup. More precisely, both (continuous and discrete time) results are based on solving an "abstract" linear program, see [9] and [24] for more details. However, the continuous time setup uses infinitely many constraints in contrast to a finite number of constraints in the discrete time case. This directly leads to two conjectures: the continuous time estimates should always yield an upper bound for their discrete time counterparts and the finer the discretization given by Definition 3.1 is, i.e., the larger $k$ is chosen, the closer the discrete time estimates should be to the continuous time ones. In Subsection 3.1, we prove these conjectures. In particular, we even show that the discrete time estimates actually converge to the continuous time ones for $k$ tending to infinity and thereby close the gap observed in [25]. Furthermore, for iterative refinements, the convergence is proven to be monotone.

In order to establish Assumption 2.5 for the various parameters $k$, i.e., the discrete time counterpart to Assumption 2.2, the time axis has to be rescaled appropriately. Here, we emphasize the contribution of using a multistep feedback which allows for employing the discrete time results without changing the underlying continuous time prediction and control horizon.

3.1. Main Results. In this section, for a given Discretization $\mathcal{D}$ according to Definition 3.1 with $k_{j} \rightarrow \infty$ for $j$ approaching infinity, the limit of the corresponding sequence of suboptimality estimates resulting from Theorem 2.6 is calculated. In particular, we show that this limit coincides with the suboptimality index of Theorem 2.3 dealing with the continuous time setting. Furthermore, if an iterative refinement is considered, this convergence is monotone which implies that the continuous time estimate represents an upper bound and that using finer discretizations improves the corresponding stability estimates. These results clarify the connection between the approaches pursued in $[9,10]$ for discrete time systems and their continuous time analogon from [24].

TheOrem 3.2. Let a discretization $\mathcal{D}=\left(\mathcal{D}_{j}\right)_{j \in \mathbb{N}_{0}}=\left(k_{j}, \tau_{j}, N_{j}, m_{j}, \sigma_{j}\right)_{j \in \mathbb{N}_{0}}$ according to Definition 3.1 with $N \in \mathbb{N}_{\geq 2}$ and $m \in\{1,2, \ldots, N-1\}$ be given. Furthermore, suppose that, for each discretization parameter $\tau_{j}, j \in \mathbb{N}_{0}$, Assumption 
2.5 is satisfied with decay rate $\sigma_{j}$ and overshoot $C \geq 1$. Furthermore, we define the expression depending on the parameter $k \in \mathbb{N}_{0}$

$$
\gamma_{i, k}:=C \sum_{n=0}^{i-1} \sigma_{k}^{n}=C \sum_{n=0}^{i-1}\left(\sigma^{1 / k}\right)^{n}=\frac{C\left(1-\sigma^{i / k}\right)}{1-\sigma^{1 / k}}
$$

and denote $\alpha_{N, m}$ from (2.10) based on $C$ and $\sigma_{k}=\sigma_{0}^{1 / k}=\sigma^{1 / k}$ by $\alpha_{k N, k m}(k)$, i.e., the argument $k$ indicates that $\gamma_{i}$ is replaced by $\gamma_{i, k}$ in (2.10). Then, we get

$$
\lim _{j \rightarrow \infty} \alpha_{k_{j} N, k_{j} m}\left(k_{j}\right)=1-\frac{\left(\frac{1-\sigma^{m}}{\sigma^{m}}\right)^{1 / C}}{\left(\frac{1-\sigma^{N}}{\sigma^{N}}\right)^{1 / C}-\left(\frac{1-\sigma^{m}}{\sigma^{m}}\right)^{1 / C}} \cdot \frac{\left(\frac{1-\sigma^{N-m}}{\sigma^{N-m}}\right)^{1 / C}}{\left(\frac{1-\sigma^{N}}{\sigma^{N}}\right)^{1 / C}-\left(\frac{1-\sigma^{N-m}}{\sigma^{N-m}}\right)^{1 / C}}
$$

if the sequence $\left(k_{j}\right)_{j \in \mathbb{N}_{0}} \subseteq \mathbb{N}$ satisfies $k_{j} \rightarrow \infty$ for $j$ tending to infinity. If, in addition, Assumption 2.2 holds with the same overshoot constant $C$ and such that the decay rate satisfies the condition $\sigma=\sigma_{0}=e^{-\mu \tau_{0}} \in(0,1)$, then we get

$$
\lim _{j \rightarrow \infty} \alpha_{k_{j} N, k_{j} m}\left(k_{j}\right)=\alpha_{T, \delta}
$$

with $\alpha_{T, \delta}$ from Formula (2.6), $\delta:=m \tau$ and $T:=N \tau$ for the continuous time control and prediction horizon, respectively.

Proof. We begin with proving the claimed convergence, i.e., (3.2) and (3.3). To this end, for $k_{j} \in\left(k_{j}\right)_{j \in \mathbb{N}_{0}}$, we rewrite

$$
\frac{\prod_{i=k_{j} m+1}^{k_{j} N}\left(\gamma_{i, k_{j}}-1\right)}{\prod_{i=k_{j} m+1}^{k_{j} N} \gamma_{i, k_{j}}-\prod_{i=k_{j} m+1}^{k_{j} N}\left(\gamma_{i, k_{j}}-1\right)}=\left[\prod_{i=k_{j} m+1}^{k_{j} N}\left(\frac{\gamma_{i, k_{j}}}{\gamma_{i, k_{j}}-1}-1\right)\right]^{-1} .
$$

Considering the limit of the right hand side w.r.t. $j$ and applying Lemma 3.6 yields

$$
\begin{aligned}
\lim _{j \rightarrow \infty} \frac{\prod_{i=k_{j} m+1}^{k_{j} N}\left(\gamma_{i, k_{j}}-1\right)}{\prod_{i=k_{j} m+1}^{k_{j} N} \gamma_{i, k_{j}}-\prod_{i=k_{j} m+1}^{k_{j} N}\left(\gamma_{i, k_{j}}-1\right)} & =\left[\frac{\left(\frac{1-\sigma^{N}}{\sigma^{N}}\right)^{1 / C}}{\left(\frac{1-\sigma^{m}}{\sigma^{m}}\right)^{1 / C}}-1\right]^{-1} \\
& =\frac{\left(\frac{1-\sigma^{m}}{\sigma^{m}}\right)^{1 / C}}{\left(\frac{1-\sigma^{N}}{\sigma^{N}}\right)^{1 / C}-\left(\frac{1-\sigma^{m}}{\sigma^{m}}\right)^{1 / C}} .
\end{aligned}
$$

Repeating this line of arguments for the second factor of the subtrahend in the formula for $\alpha_{k_{j} N, k_{j} m}\left(k_{j}\right)$ and combining the result with (3.4) shows (3.2). In order to complete the proof, we have to establish equality of the right hand sides of (3.2) and (3.3). Using the definitions of $\sigma$ and $\delta$ we obtain

$$
\left(\frac{1-\sigma^{m}}{\sigma^{m}}\right)^{1 / C}=\left(\frac{1-e^{-\mu m \tau}}{e^{-\mu m \tau}}\right)^{1 / C}=\left(\frac{1-e^{-\mu \delta}}{e^{-\mu \delta}}\right)^{1 / C}=\left(e^{\mu \delta}-1\right)^{1 / C} .
$$

Hence, taking the definition of $T$ into account, repeating this argumentation and pluggig the resulting expressions in (3.2) allows for concluding the assertion. $\square$

Theorem 3.2 shows the connection between the unconstrained MPC setup for continuous time systems presented in Section 2.1 and the discrete time setting in Section 2.2. The continuous time results can be interpreted as limit case of what performance guarantee can be achieved for sufficiently fast sampling. 
For an iterative refinement, we can additionally show monotonicity of this convergence, a result which generalizes [29, Proposition 5.13] to arbitrary iterative refinements.

Proposition 3.3. Let the sequence $\left(k_{j}\right)_{j \in \mathbb{N}_{0}} \subset \mathbb{N}$ be an iterative refinement according to Definition 3.1. Then, the sequence $\left(\alpha_{k_{j} N, k_{j} m}\left(k_{j}\right)\right)_{j \in \mathbb{N}_{0}}$ defined in Theorem 3.2 is monotonically increasing in $j$.

Proof. Rewriting Formula (2.10) analogously to (3.4) yields

$$
\alpha_{k_{j} N, k_{j} m}\left(k_{j}\right)=\left[\prod_{i=k_{j} m+1}^{k_{j} N} \frac{\gamma_{i, k_{j}}}{\gamma_{i, k_{j}}-1}-1\right]^{-1} \cdot\left[\prod_{i=k_{j}(N-m)+1}^{k_{j} N} \frac{\gamma_{i, k_{j}}}{\gamma_{i, k_{j}}-1}-1\right]^{-1} .
$$

Hence, showing that each of the two factors on the right hand side of this representation of $\alpha_{k_{j} N, k_{j} m}\left(k_{j}\right)$ is monotonically decreasing with respect to $j$ is sufficient in order to prove the claimed monotonicity. This is, in turn, equivalent to the condition that the products $\prod_{i=k_{j} m+1}^{k_{j} N} \frac{\gamma_{i, k_{j}}}{\gamma_{i, k_{j}}-1}$ and $\prod_{i=k_{j}(N-m)+1}^{k_{j} N} \frac{\gamma_{i, k_{j}}}{\gamma_{i, k_{j}}-1}$ are both monotonically increasing in $j$. Since an iterative refinement is considered, $\kappa_{j}:=\frac{k_{j+1}}{k_{j}} \in \mathbb{N}$ holds. Hence, showing the following inequality allows to conclude the assertion

$$
\prod_{i=k_{j+1} m+1}^{k_{j+1} N} \frac{\gamma_{i, k_{j+1}}}{\gamma_{i, k_{j+1}}-1}=\prod_{i=k_{j} m+1}^{k_{j} N} \prod_{\varrho=1}^{\kappa_{j}} \frac{\gamma_{\kappa_{j}(i-1)+\varrho, \kappa_{j} k_{j}}}{\gamma_{\kappa_{j}(i-1)+\varrho, \kappa_{j} k_{j}}-1} \geq \prod_{i=k_{j} m+1}^{k_{j} N} \frac{\gamma_{i, k_{j}}}{\gamma_{i, k_{j}}-1} .
$$

In order to prove this inequality, it is sufficient to deduce the inequality

$$
\left(\gamma_{i, k_{j}}-1\right) \prod_{\varrho=1}^{\kappa_{j}} \gamma_{\kappa_{j}(i-1)+\varrho, \kappa_{j} k_{j}} \geq \gamma_{i, k_{j}} \prod_{\varrho=1}^{\kappa_{j}}\left(\gamma_{\kappa_{j}(i-1)+\varrho, \kappa_{j} k_{j}}-1\right)
$$

for each $i \in\left\{k_{j} m+1, k_{j} m+2, \ldots, k_{j} N\right\}$, which corresponds to showing (3.5) componentwise. Splitting up the left hand side of (3.6) and factoring out $\gamma_{i, k_{j}}$ leads to

$$
\prod_{\varrho=1}^{\kappa_{j}} \gamma_{\kappa_{j}(i-1)+\varrho, \kappa_{j} k_{j}} \leq \gamma_{i, k_{j}}\left[\prod_{\varrho=1}^{\kappa_{j}} \gamma_{\kappa_{j}(i-1)+\varrho, \kappa_{j} k_{j}}-\prod_{\varrho=1}^{\kappa_{j}}\left(\gamma_{\kappa_{j}(i-1)+\varrho, \kappa_{j} k_{j}}-1\right)\right] .
$$

According to Lemma 6.1 in Section 6, see also [29, Lemma 3.21], the term in square brackets is equal to

$$
\sum_{\varrho=1}^{\kappa_{j}}\left(\prod_{\mu=1}^{\varrho-1} \gamma_{\kappa_{j}(i-1)+\mu, \kappa_{j} k_{j}} \prod_{\mu=\varrho+1}^{\kappa_{j}}\left(\gamma_{\kappa_{j}(i-1)+\mu, \kappa_{j} k_{j}}-1\right)\right) .
$$

Hence, since $C \geq 1$ holds, showing

$$
\prod_{\varrho=1}^{\kappa_{j}} \gamma_{\kappa_{j}(i-1)+\varrho, \kappa_{j} k_{j}} \leq \gamma_{i, k_{j}}\left[\sum_{\varrho=1}^{\kappa_{j}}\left(\prod_{\mu=1}^{\varrho-1} \gamma_{\kappa_{j}(i-1)+\mu, \kappa_{j} k_{j}} \prod_{\mu=\varrho+1}^{\kappa_{j}}\left(\gamma_{\kappa_{j}(i-1)+\mu, \kappa_{j} k_{j}}-C\right)\right)\right]
$$

is sufficient in order to ensure (3.7). In (3.8) each summand contains the factor $C^{\kappa_{j}}$. Hence, reducing this inequality by $C^{\kappa_{j}}$ and, then, applying Lemma 6.1 once more in 
order to "undo" the transformation carried out leads to (3.6) with $\gamma_{i, k}$ replaced by $\gamma_{i, k} / C$, i.e.,

$$
\left(\frac{\gamma_{i, k_{j}}}{C}-1\right) \prod_{\varrho=1}^{\kappa_{j}} \frac{\gamma_{\kappa_{j}(i-1)+\varrho, \kappa_{j} k_{j}}}{C} \geq \frac{\gamma_{i, k_{j}}}{C} \prod_{\varrho=1}^{\kappa_{j}}\left(\frac{\gamma_{\kappa_{j}(i-1)+\varrho, \kappa_{j} k_{j}}}{C}-1\right) .
$$

Then, using the definition $\gamma_{i, k}=\frac{C\left(1-\sigma^{i / k}\right)}{1-\sigma^{1 / k}}$ from Theorem 3.2 and simple manipulations show that Inequality (3.9) holds with equality, which in turn implies (3.8) - an observation which completes the proof. $\square$

A direct consequence of Proposition 3.3 is that the performance estimate resulting from applying Theorem 2.3 represents an upper bound on the suboptimality bounds obtained in the discrete time setting.

Corollary 3.4. Let the assumption of Theorem 3.2 hold. Then, for each $k \in$ $\left(k_{j}\right)_{j \in \mathbb{N}_{0}}$, the right hand side of (3.2) is an upper bound on $\alpha_{k N, k m}(k), k \in \mathbb{N}_{0}$, i.e.,

$$
\alpha_{k N, k m}(k) \leq 1-\frac{\left(\frac{1-\sigma^{m}}{\sigma^{m}}\right)^{1 / C}}{\left(\frac{1-\sigma^{N}}{\sigma^{N}}\right)^{1 / C}-\left(\frac{1-\sigma^{m}}{\sigma^{m}}\right)^{1 / C}} \cdot \frac{\left(\frac{1-\sigma^{N-m}}{\sigma^{N-m}}\right)^{1 / C}}{\left(\frac{1-\sigma^{N}}{\sigma^{N}}\right)^{1 / C}-\left(\frac{1-\sigma^{N-m}}{\sigma^{N-m}}\right)^{1 / C}} .
$$

Proof. Since for each $k \in \mathbb{N}_{0}$ an iterative refinement sequence given by $\left(k_{j}\right)_{j \in \mathbb{N}_{0}}$ with $k_{j}:=2^{j} k$ can be constructed, the proof follows directly from the Limit (3.2) shown in Theorem 3.2 and the monotonicity in Proposition 3.3.

The assertions of Theorem 3.2, Proposition 3.3, and Corollary 3.4 are illustrated in Figure 3.1 for an iterative refinement. The discrete time estimates approximate their continuous time counterpart already after very few refinements astonishingly well. This observation is supported by the illustration drawn on the right which shows that the discretization error decays exponentially.
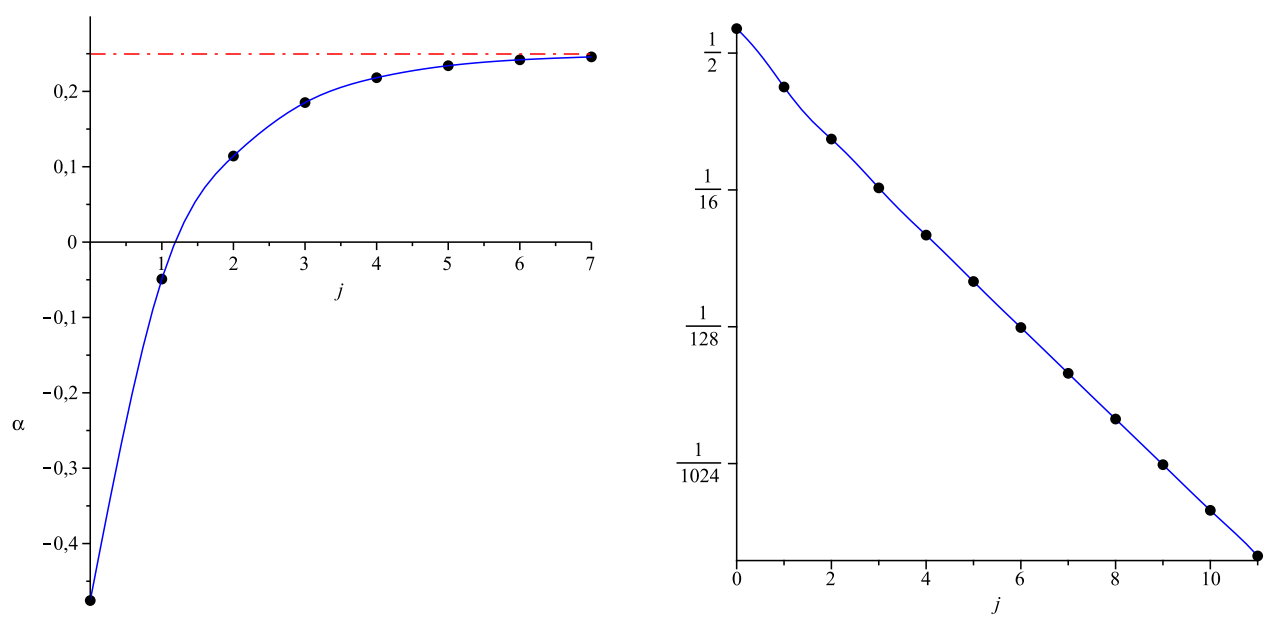

FIG. 3.1. The figure on the left dipicts the monotone convergence of the sequence of suboptimality estimates $\left(\alpha_{k_{j} N, k_{j} m}\left(k_{j}\right)\right)_{j \in \mathbb{N}_{0}}$ for $k_{j}=2^{j}$ to the limit computed in Theorem 3.2. On the right, the deviations from the theoretically obtained upper bound are depicted. This error decays exponentially in the iteration index $j$. Here, we choose $N=4, m=1, \tau=1, C=2$, and $\sigma=0.5$.

Summarizing, we showed that stability conditions and performance guarantees obtained in the continuous time case represent an upper bound for their discrete time 
counterparts. However, the continuous time results can be arbitrarily well approximated for a sufficiently small sampling period $\tau$ in combination with a multistep feedback law. Hence, the discrete time results can be used in order to investigate the trade-off between slow sampling and guaranteed performance: typically sampled-data systems use piecewise constant control functions, i.e., the control value only changes at the sampling instances. Hence, in order to approximate the continuous time solution sufficiently fast sampling is, in general, required, cf. [20]. In particular, a larger discretization parameter $k$ allows also to approximate the control function $\left.u(\cdot)\right|_{0, \delta}$ more precisely, cf. [1]. For such a setup, the discrete time results of Theorem 2.6 are also applicable. The continuous time results of Theorem 2.3 can provide an upper bound on the achievable guaranteed performance for infinitely fast sampling. In conclusion, the results in this work give insight into the trade off between slow sampling and good performance.

3.2. Auxiliary Results for the Proof of Theorem 3.2. This subsection presents intermediate results for the proof of Theorem 3.2. We start with a technical lemma whose proof is based on a simple Taylor series expansion.

Lemma 3.5. Let a parameter $s \in(0,1)$ and a constant $c \in \mathbb{R}_{>0}$ be given such that $c-1+s>0$ holds. Then, for each sequence $\left(n_{i}\right)_{i \in \mathbb{N}} \subset \mathbb{N}$ satisfying $n_{i} \rightarrow \infty$ for $i \rightarrow \infty$, we get the convergence

$$
\left(1+\frac{1-s^{\frac{1}{n_{i}}}}{c-\left(1-s^{\frac{1}{n_{i}}}\right)}\right)^{n_{i}} \longrightarrow\left(s^{-1}\right)^{\frac{1}{c}} \quad \text { for } i \rightarrow \infty .
$$

Proof. We define the analytic function $f: \mathbb{R}_{\leq 1} \rightarrow \mathbb{R}$ and calculate its first derivative

$$
\begin{aligned}
f(x) & :=\frac{1-s^{x}}{c-\left(1-s^{x}\right)}=\frac{1-e^{x \ln s}}{c-\left(1-e^{x \ln s}\right)} \\
f^{\prime}(x) & =\frac{-c \ln s \cdot s^{x}}{\left(c-\left(1-s^{x}\right)\right)^{2}}=\frac{-c \ln s \cdot e^{x \ln s}}{\left(c-\left(1-e^{x \ln s}\right)\right)^{2}} .
\end{aligned}
$$

Furthermore, we point out that the norm of the second derivative $f^{\prime \prime}(\cdot)$ is uniformly bounded on the interval $[0,1]$, i.e., there exists a constant $M \in \mathbb{R}_{>0}$ such that $\sup _{x \in[0,1]}\left|f^{\prime \prime}(x)\right| \leq M$. In addition, $f(0)=0$ and $f^{\prime}(0)=-(\ln s) / c$ hold. Hence, for each element $n \in\left(n_{i}\right)_{i \in \mathbb{N}}$, using the Taylor series expansion of $f(1 / n)$ at $x=0$, cf. [15, Chapter XIII], yields the existence of a real number $\xi_{n} \in(0,1 / n)$ such that

$$
f(1 / n)=f(0)+\frac{1}{n} f^{\prime}(0)+\sum_{j=2}^{\infty}\left(\frac{1}{n}\right)^{j} \frac{f^{(j)}(0)}{j !}=-\frac{\ln s}{c n}+\frac{f^{\prime \prime}\left(\xi_{n}\right)}{2 n^{2}} .
$$

Since $\xi_{n} \in(0,1 / n) \subseteq(0,1),\left|f^{\prime \prime}\left(\xi_{n}\right)\right| \leq M$ independently of the considered element $n$. Moreover, for an arbitrarily chosen constant $\varepsilon>0$ and sufficiently large $n$ (which holds for all $n_{i} \in\left(n_{i}\right)_{i \in \mathbb{N}}$ with sufficiently large index $\left.i\right),\left(1+f^{\prime}(0) / n\right)^{n} \leq e^{f^{\prime}(0)}+\varepsilon$ holds because $\left(1+f^{\prime}(0) / n_{i}\right)^{n_{i}} \rightarrow e^{f^{\prime}(0)} \in[1, \infty)$ for $i$ tending to infinity. These prelimary considerations enable us to deduce the following estimate which is essential 
in order to conclude the assertion. Since $1+\frac{1}{n} f^{\prime}(0) \geq 1$, we get

$$
\begin{aligned}
0 & \leq \sum_{j=1}^{n}\left(\begin{array}{l}
n \\
j
\end{array}\right)\left(1+\frac{f^{\prime}(0)}{n}\right)^{n-j}\left(\frac{\left|f^{\prime \prime}\left(\xi_{n}\right)\right|}{2 n^{2}}\right)^{j} \\
& =\sum_{j=1}^{n}\left(\frac{\left|f^{\prime \prime}\left(\xi_{n}\right)\right|}{2}\right)^{j} \frac{1}{j !} \underbrace{\frac{n(n-1)(n-2) \cdots(n-j+1)}{n}}_{\leq 1} \underbrace{\left(1+\frac{f^{\prime}(0)}{n}\right)^{n-j}}_{\leq\left(1+\frac{1}{n} f^{\prime}(0)\right)^{n}} \underbrace{\left(\frac{1}{n}\right)^{j}}_{\leq \frac{1}{n}} \\
& <\left(e^{f^{\prime}(0)}+\varepsilon\right) \frac{1}{n} \sum_{j=0}^{n} \frac{(M / 2)^{j}}{j !}<\left(e^{f^{\prime}(0)}+\varepsilon\right) \frac{e^{\frac{M}{2}}}{n} \stackrel{n \rightarrow \infty}{\longrightarrow} 0 .
\end{aligned}
$$

Taking this into account and carrying out a binomial expansion, cf. [15, p.466], we obtain

$$
\left(1+f\left(1 / n_{i}\right)\right)^{n_{i}} \stackrel{(3.11)}{=}\left(1+f^{\prime}(0) / n_{i}\right)^{n_{i}}+\sum_{j=1}^{n_{i}}\left(\begin{array}{c}
n_{i} \\
j
\end{array}\right)\left(1+\frac{f^{\prime}(0)}{n_{i}}\right)^{n_{i}-j}\left(\frac{f^{\prime \prime}\left(\xi_{n_{i}}\right)}{2 n_{i}^{2}}\right)^{j} \stackrel{i \rightarrow \infty}{\longrightarrow} e^{f^{\prime}(0)} .
$$

In view of the definition of $f(\cdot), e^{f^{\prime}(0)}=e^{-(\ln s) / c}=\left(s^{-1}\right)^{\frac{1}{c}}$ completes the proof. $\square$

The next lemma embodies the cornerstone needed in order to prove Theorem 3.2.

Lemma 3.6. Let $\sigma \in(0,1), C \geq 1, N \in \mathbb{N}_{\geq 2}$, and $m \in\{1,2, \ldots, N-1\}$ be given and define $\sigma_{k}:=\sqrt[k]{\sigma}$. Then, for $\gamma_{i, k}=C \sum_{n=0}^{i-1} \sigma_{k}^{n}$ and a sequence $\left(k_{j}\right)_{j \in \mathbb{N}_{0}} \subset \mathbb{N}$ satisfying $k_{j} \rightarrow \infty$ for $j$ tending to infinity, the following convergence holds:

$$
\prod_{i=m k_{j}+1}^{N k_{j}} \frac{\gamma_{i, k_{j}}}{\gamma_{i, k_{j}}-1} \longrightarrow\left(\sigma^{-(N-m)} \cdot \frac{1-\sigma^{N}}{1-\sigma^{m}}\right)^{\frac{1}{C}} \quad \text { for } j \rightarrow \infty .
$$

Proof. Let the sequence $\left(k_{j}\right)_{j \in \mathbb{N}_{0}}$ be an iterative refinement according to Definition 3.1. This restriction is justified in view of Remark 3.7 in which the necessary modifications needed for arbitrary discretization sequences are briefly sketched. Furthermore, let $k^{\star}$ denote an arbitrary but fixed element of the parameter sequence $\left(k_{j}\right)_{j \in \mathbb{N}_{0}}$. Then, for each $k \in\left(k_{j}\right)_{j \in \mathbb{N}_{0}}$ satisfying $k \geq k_{j^{\star}}$ the left hand side of (3.12) can be rewritten as

$$
\begin{aligned}
\prod_{i=m k+1}^{N k} \frac{\gamma_{i, k}}{\gamma_{i, k}-1} & =\prod_{\nu=m}^{N-1} \prod_{i=1}^{k}\left(1+\frac{1-\sigma^{\frac{1}{k}}}{C\left[1-\sigma^{\nu} \sigma^{\frac{i}{k}}\right]-1+\sigma^{\frac{1}{k}}}\right) \\
& =\prod_{\nu=m}^{N-1} \prod_{l=0}^{k^{\star}-1} \prod_{i=1}^{k / k^{\star}}\left(1+\frac{1-\sigma^{\frac{1}{k}}}{C\left[1-\sigma^{\nu+\frac{l}{k^{\star}}} \sigma^{\frac{i}{k}}\right]-1+\sigma^{\frac{1}{k}}}\right)
\end{aligned}
$$

The denominator of the involved factors, i.e., $C\left(1-\sigma^{\nu+\frac{l}{k^{\star}}} \sigma^{\frac{i}{k}}\right)-\left(1-\sigma^{\frac{1}{k}}\right)$, is (strictly) greater than $C\left(1-\sigma^{\nu}\right)-(1-\sigma) \geq(C-1)(1-\sigma)$ and, thus, (strictly) positive. Hence, dividing the respective denominators increases the corresponding fractions, i.e., leads to an estimate from above. Bearing this in mind, yields

$$
\begin{aligned}
& \left(1+\frac{1-\sigma^{\frac{1}{k}}}{C\left[1-\sigma^{\nu+\frac{l+1}{k^{\star}}}\right]-1+\sigma^{\frac{1}{k}}}\right) \leq\left(1+\frac{1-\sigma^{\frac{1}{k}}}{C\left[1-\sigma^{\nu+\frac{l}{k^{\star}}} \sigma^{\frac{i}{k}}\right]-1+\sigma^{\frac{1}{k}}}\right) \\
& <\left(1+\frac{1-\sigma^{\frac{1}{k}}}{C\left[1-\sigma^{\nu+\frac{l}{k^{*}}}\right]-1+\sigma^{\frac{1}{k}}}\right)
\end{aligned}
$$


for $i \in\left\{1,2, \ldots, k / k^{\star}\right\}$. Note that the lower and upper estimate do not depend on the index $i$ which motivates, for $\nu \in\{m, m+1, \ldots, N-1\}$ and $l \in\left\{0,1, \ldots, k^{\star}-1\right\}$, the definitions

$$
\begin{aligned}
& \bar{g}_{\nu, l}\left(k ; k^{\star}\right):=\left(1+\frac{1-\sigma^{\frac{1}{k}}}{C\left[1-\sigma^{\nu+\frac{l}{k^{\star}}}\right]-1+\sigma^{\frac{1}{k}}}\right)^{\frac{k}{k^{\star}}}, \\
& \underline{g}_{\nu, l}\left(k ; k^{\star}\right):=\left(1+\frac{1-\sigma^{\frac{1}{k}}}{C\left[1-\sigma^{\nu+\frac{l+1}{k^{\star}}}\right]-1+\sigma^{\frac{1}{k}}}\right)^{\frac{k}{k^{\star}}} .
\end{aligned}
$$

Carrying out the transformation $k_{j}=k^{\star} \tilde{k}_{j}$, i.e., $k_{j} / k^{\star}=\tilde{k}_{j}$, for $k_{j} \geq k^{\star}$ enables us to apply Lemma 3.5 with $s:=\sigma^{1 / k^{\star}}$ and $c:=C\left[1-\sigma^{\nu+l / k^{\star}}\right] \geq 1-\sigma^{\nu}>1-s\left(k^{\star} \geq 1\right)$ to $\bar{g}_{\nu, l}\left(\cdot ; k^{\star}\right)$ which provides

$$
\bar{g}_{\nu, l}\left(k_{j} ; k^{\star}\right) \stackrel{j \rightarrow \infty}{\longrightarrow}\left(s^{-1}\right)^{\frac{1}{c}}=\left(\sigma^{-\frac{1}{k^{\star}}}\right)^{\frac{1}{c}}=\left(\sigma^{-\frac{1}{k^{\star}}}\right)^{\frac{1}{C\left[1-\sigma^{\left.\nu+l / k^{\star}\right]}\right.}}=: \bar{g}_{\nu, l}^{\star}\left(k^{\star}\right) .
$$

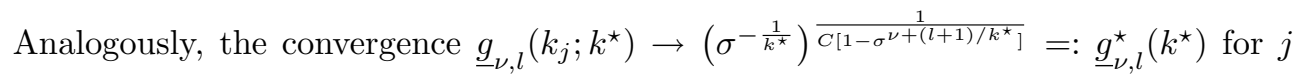
approaching infinity follows. Note that these formulas confirm $0<\underline{g}_{\nu, l}^{\star}<\bar{g}_{\nu, l}^{\star}<\infty$. We continue our examination of these auxiliary approximations. To this end, we define

$$
\overline{\mathcal{G}}\left(k^{\star}\right):=\lim _{j \rightarrow \infty} \prod_{\nu=m}^{N-1} \prod_{l=0}^{k^{\star}-1} \bar{g}_{\nu, l}\left(k_{j} ; k^{\star}\right) \quad \text { and } \quad \underline{\mathcal{G}}\left(k^{\star}\right):=\lim _{j \rightarrow \infty} \prod_{\nu=m}^{N-1} \prod_{l=0}^{k^{\star}-1} \underline{g}_{\nu, l}\left(k_{j} ; k^{\star}\right) .
$$

Note that the above computations ensure that $\overline{\mathcal{G}}\left(k^{\star}\right)$ and $\underline{\mathcal{G}}\left(k^{\star}\right)$ are located in the open interval $(0, \infty)$ and that, for each $j \in \mathbb{N}_{0}$ satisfying $k_{j} \geq k^{\star}$, the following inequality holds

$$
\underline{\mathcal{G}}\left(k^{\star}\right) \leq \prod_{i=m k_{j}+1}^{N k_{j}} \frac{\gamma_{i, k_{j}}}{\gamma_{i, k_{j}}-1} \leq \overline{\mathcal{G}}\left(k^{\star}\right) .
$$

Clearly, choosing a larger element $k^{\star}$ refines the approximation. Hence, $\overline{\mathcal{G}}\left(k^{\star}\right)$ is monotonically decreasing and $\underline{\mathcal{G}}\left(k^{\star}\right)$ is monotonically increasing with respect to $k^{\star}$ which, since $\underline{\mathcal{G}}\left(k^{\star}\right)<\overline{\mathcal{G}}\left(k^{\star}\right)$ holds for all $k^{\star} \in \mathbb{N}$, guarantees the existence of the respective limits for $k^{\star}$ approaching infinity and $\lim _{k^{\star} \rightarrow \infty} \underline{\mathcal{G}}\left(k^{\star}\right) \leq \lim _{k^{\star} \rightarrow \infty} \overline{\mathcal{G}}\left(k^{\star}\right)$. Indeed, this inequality turns out to be an equality:

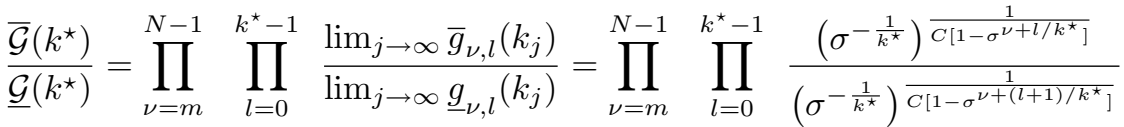

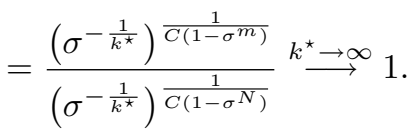

This allows for drawing conclusions on the limit of the left hand side of (3.12). For $k^{\star}$ tending to infinity, each of the two introduced approximations converges to the same finite limit. Hence, it remains to verify that the expression specified in the right hand side of (3.12) equals the limit of the introduced approximations. To this end, 
we exploit the specific form of the limits $\bar{g}_{\nu, l}^{\star}\left(k^{\star}\right)$ and $\underline{g}_{\nu, l}^{\star}\left(k^{\star}\right)$. In particular, Lemma 3.5 yields

$$
\begin{aligned}
& \left(\bar{g}_{\nu, l}^{\star}\right)^{C}=\left(\sigma^{-\frac{1}{k^{\star}}}\right)^{\frac{1}{1-\sigma^{\nu+l / k^{\star}}}}=\lim _{j \rightarrow \infty} \prod_{i=1}^{k_{j} / k^{\star}}\left(1+\frac{1-\sigma^{1 / k_{j}}}{1-\sigma^{j+l / k^{\star}}-1+\sigma^{1 / k_{j}}}\right) \quad \text { and } \\
& \left(\underline{g}_{\nu, l}^{\star}\right)^{C}=\left(\sigma^{-\frac{1}{k^{\star}}}\right)^{\frac{1}{1-\sigma^{\nu+(l+1) / k^{\star}}}}=\lim _{j \rightarrow \infty} \prod_{i=1}^{k_{j} / k^{\star}}\left(1+\frac{1-\sigma^{1 / k_{j}}}{1-\sigma^{j+(l+1) / k^{\star}}-1+\sigma^{1 / k_{j}}}\right) .
\end{aligned}
$$

This allows for elaborating the following chain of inequalities, which resembles the structure of (3.14). Again, we use $k \in\left(k_{j}\right)_{j \in \mathbb{N}_{0}}$ satisfying $k \geq k^{\star}$ in order to avoid technical difficulties

$$
\prod_{i=1}^{k / k^{\star}}\left(1+\frac{1-\sigma^{1 / k}}{\sigma^{1 / k}-\sigma^{\nu+(l+1) / k^{\star}}}\right) \leq \prod_{i=1}^{k / k^{\star}}\left(1+\frac{1-\sigma^{1 / k}}{\sigma^{1 / k}-\sigma^{\nu+l / k^{\star}} \sigma^{i / k}}\right)<\prod_{i=1}^{k / k^{\star}}\left(1+\frac{1-\sigma^{1 / k}}{\sigma^{1 / k}-\sigma^{\nu+l / k^{\star}}}\right) .
$$

However, in contrast to (3.14), we are able to deal with the term representing the core of this expression using an argument similar to those applied to telescoping series

$$
\begin{aligned}
\prod_{i=1}^{k / k^{\star}}\left(1+\frac{1-\sigma^{1 / k}}{\sigma^{1 / k}-\sigma^{\nu+l / k^{\star}} \sigma^{i / k}}\right) & =\prod_{i=1}^{k / k^{\star}} \frac{1-\sigma^{\nu+l / k^{\star}} \sigma^{i / k}}{\sigma^{1 / k}\left(1-\sigma^{\nu+l / k^{\star}} \sigma^{(i-1) / k}\right)} \\
& =\sigma^{-1 / k^{\star}} \cdot \frac{1-\sigma^{\nu+(l+1) / k^{\star}}}{1-\sigma^{\nu+l / k^{\star}}} .
\end{aligned}
$$

Hence, using these prelimary considerations leads to

$$
\begin{aligned}
\underline{\mathcal{G}}\left(k^{\star}\right) \leq \prod_{\nu=m}^{N-1} \prod_{l=0}^{k^{\star}-1}\left[\sigma^{-\frac{1}{k^{\star}}} \frac{1-\sigma^{\nu+\frac{l+1}{k^{\star}}}}{1-\sigma^{\nu+\frac{l}{k^{\star}}}}\right]^{\frac{1}{C}} & =\prod_{\nu=m}^{N-1}\left[\sigma^{-1} \frac{1-\sigma^{\nu+1}}{1-\sigma^{\nu}}\right]^{\frac{1}{C}} \\
& =\left[\sigma^{-(N-m)} \frac{1-\sigma^{N}}{1-\sigma^{m}}\right]^{\frac{1}{C}} \leq \overline{\mathcal{G}}\left(k^{\star}\right)
\end{aligned}
$$

for each $k^{\star}$ which, since $\underline{\mathcal{G}}\left(k^{\star}\right) \leq \lim _{k^{\star} \rightarrow \infty} \underline{\mathcal{G}}\left(k^{\star}\right)=\lim _{k^{\star} \rightarrow \infty} \overline{\mathcal{G}}\left(k^{\star}\right) \leq \overline{\mathcal{G}}\left(k^{\star}\right)$ holds, implies that the involved limits coincide with the deduced bound, i.e.,

$$
\lim _{k^{\star} \rightarrow \infty} \underline{\mathcal{G}}\left(k^{\star}\right)=\left[\sigma^{-(N-m)} \frac{1-\sigma^{N}}{1-\sigma^{m}}\right]^{\frac{1}{C}}=\lim _{k^{\star} \rightarrow \infty} \overline{\mathcal{G}}\left(k^{\star}\right) .
$$

Summarizing these computations provides

$$
\begin{aligned}
{\left[\sigma^{-(N-m)} \frac{1-\sigma^{N}}{1-\sigma^{m}}\right]^{\frac{1}{C}} } & =\lim _{k^{\star} \rightarrow \infty} \underline{\mathcal{G}}\left(k^{\star}\right) \leq \lim _{j \rightarrow \infty} \prod_{i=k_{j} m+1}^{k_{j} N} \frac{\gamma_{i, k_{j}}}{\gamma_{i, k_{j}}-1} \\
& \leq \lim _{k^{\star} \rightarrow \infty} \overline{\mathcal{G}}\left(k^{\star}\right)=\left[\sigma^{-(N-m)} \frac{1-\sigma^{N}}{1-\sigma^{m}}\right]^{\frac{1}{C}}
\end{aligned}
$$

and, thus, ensures (3.12), i.e., the assertion.

REMARK 3.7. The restriction to iterative refinements is not necessary in order to prove Lemma 3.6 but allows the reader to concentrate on the essential steps without 
being distracted by technical details. Since $k_{j} \rightarrow \infty$ for $j$ tending to infinity, for each index $j^{\star}$ determining an element $k^{\star}=k_{j^{\star}}$ an index $\bar{j} \geq j^{\star}$ exists such that $k_{j} \geq k_{j^{\star}}$ holds for all $j \geq \bar{j}$ for the parameter sequence $\left(k_{j}\right)_{j \in \mathbb{N}_{0}}$. For each $k$ satisfying this condition, a switching index $\tilde{k}^{\star}:=k \bmod k^{\star}$ can be defined. Then, the product

$$
\prod_{l=0}^{k^{\star}-1} \prod_{i=1}^{k / k^{\star}}\left(1+\frac{1-\sigma^{\frac{1}{k}}}{C\left[1-\sigma^{\nu+\frac{l}{k}{ }^{\star}} \sigma^{\frac{i}{k}}\right]-1+\sigma^{\frac{1}{k}}}\right)
$$

from (3.13) is replaced by

$\prod_{l=0}^{\tilde{k}^{\star}-1} \prod_{i=1}^{\left\lceil k / k^{\star}\right\rceil}\left(1+\frac{1-\sigma^{\frac{1}{k}}}{C\left[1-\sigma^{\nu+\frac{l}{k^{\star}}} \sigma^{\frac{i}{k}}\right]-1+\sigma^{\frac{1}{k}}}\right) \cdot \prod_{l=\tilde{k}^{\star}}^{k^{\star}-1} \prod_{i=1}^{\left\lfloor k / k^{\star}\right\rfloor}\left(1+\frac{1-\sigma^{\frac{1}{k}}}{C\left[1-\sigma^{\nu+\frac{l}{k^{\star}}} \sigma^{\frac{i}{k}}\right]-1+\sigma^{\frac{1}{k}}}\right)$,

i.e., the involved factors are distributed such that the number of factors is either $\left\lfloor k / k^{\star}\right\rfloor$ or $\left\lceil k / k^{\star}\right\rceil$ and the total number of factors is equal to $k$. The following chain of inequalities remains unchanged, only the corresponding index range has to be adapted to the set $\left\{1,2, \ldots,\left\lceil k / k^{\star}\right\rceil\right\}$. The upper index of the product in the definitions of $\bar{g}_{\nu, l}\left(k ; k^{\star}\right)$ and $\underline{g}_{\nu, l}\left(k ; k^{\star}\right)$ depends on whether or not the index $l$ is contained in $\left[0, \tilde{k}^{\star}-\right.$ 1] for the considered argument $k$. However, since we are only interested in the limit for $k$ approaching infinity, this distinction does not play a role: looking at the proof of Lemma 3.5 shows that the assertion also holds for a sequence $\left(n_{i}\right)_{i \in \mathbb{N}_{0}} \subset \mathbb{R}_{0}^{+}$satisfying $n_{i} \rightarrow \infty$, if the exponent is, for each index $i$, randomly substituted by either $\left\lfloor n_{i}\right\rfloor$ or $\left\lceil n_{i}\right\rceil$. The remaining part of the proof of Lemma 3.6 does not require further modifications.

4. Examples. In order to illustrate our results, an example of a reaction diffusion equation taken from [3] is considered in Subsection 4.1. Subsection 4.2 gives some additional remarks on the controllability conditions introduced in Section 2.

4.1. Reaction Diffusion Equation. Let $\Omega \subset \mathbb{R}$ be an open interval. We consider a reaction diffusion equation

$$
\begin{array}{rlrlrl}
z_{t}(x, t) & =z_{x x}(x, t)-h(z(x, t))+u(x, t) & & \text { on } & & \Omega \times \mathbb{R}_{>0}, \\
z(x, t) & =0 & & \text { on } & \partial \Omega \times \mathbb{R}_{>0}
\end{array}
$$

with homogeneous Dirichlet boundary conditions, distributed control $u(\cdot, t): \Omega \rightarrow \mathbb{R}$, and continuously differentiable nonlinearity $h: \Omega \rightarrow \mathbb{R}$. The initial data are given by $z(x, 0)=z_{0}(x), x \in \Omega$. In addition, the condition $h(0)=0$ is supposed in order to ensure that the origin is an equilibrium. This semilinear parabolic partial differential equation (PDE) will be used in order to illustrate the derived theoretical results. We start off with the continuous time setting introduced in Subsection 2.1

The state space $Z$ and the set of feasible states $\mathbb{Z}$ are both equal to $\mathcal{H}^{1}(\Omega)$. Since no control constraints are imposed, the set $\mathbb{U}$ of admissible control values is given by $U=\mathcal{L}^{\infty}(\Omega)$. Our goal is to stabilize the equilibrium $z^{\star}=z^{\star}(\cdot) \equiv 0$. For existence and regularity results we refer to [4]. The stage costs $\ell: Z \times U \rightarrow \mathbb{R}_{\geq 0}$ are defined by

$$
\ell(z, u)=\left\|z-z^{\star}\right\|_{\mathcal{L}^{2}(\Omega)}^{2}+\lambda\|u\|_{\mathcal{L}^{2}(\Omega)}^{2}=\|z\|_{\mathcal{L}^{2}(\Omega)}^{2}+\lambda\|u\|_{\mathcal{L}^{2}(\Omega)}^{2}
$$

with regularization parameter $\lambda>0$. The second term penalizes the control effort used to steer the system in the desired direction. 
Next, we want to verify Assumption 2.2, i.e., we aim at showing Inequality (2.5) by determining suitable parameters $C, \sigma$. Then, a prediction horizon $T$ can be computed for which Theorem 2.3 guarantees asymptotic stability of the corresponding MPC closed-loop. To this end, the following theorem concerning the local stability behavior of the uncontrolled version of this semilinear parabolic equation is presented, cf. [4].

THEOREM 4.1. For each $\gamma \in\left(0, \lambda_{1}+h^{\prime}(0)\right)$ there exists a constant $R=R(\gamma)$ such that for all $z_{0} \in \mathcal{C}_{0}(\Omega)$ with $\left\|z_{0}\right\| \leq R$ the solution $z_{u}(\cdot, t)$ of (4.1), (4.2) with $u(\cdot, t) \equiv 0, t \in \mathbb{R}_{\geq 0}$, satisfies

$$
\left\|z_{u}(\cdot, t)\right\| \leq M\left\|z_{0}\right\| e^{-\gamma t} \quad \text { for all } t \geq 0 .
$$

Here, $\lambda_{1}=\lambda_{1}(\Omega)$ denotes the smallest eigenvalue of the differential operator $-\Delta$ in $\mathcal{H}_{0}^{1}(\Omega)$.

The constant $M$ from (4.4) is independent of $\gamma$ and the given initial state $z_{0}$. Indeed, it only depends on the used norm, e.g., $M=1$ for $\|\cdot\|_{\mathcal{L}^{2}(\Omega)}$. Furthermore, the origin is unstable for $\lambda_{1}<-h^{\prime}(0)$, cf. [4] for details. An important representative of the considered class of reaction diffusion equations is the one dimensional ChafeeInfante equation

$$
z_{t}(x, t)=z_{x x}(x, t)+\nu\left(z(x, t)-z(x, t)^{3}\right)+u(x, t),
$$

i.e., $h(z)=-\nu\left(z-z^{3}\right)$. Existence results for the optimal control problem solved in each MPC iteration can be found in [19]. For parameter $\nu=11$ and initial data $z(x, 0)=0.2 \sin (\pi x)$, the origin is unstable because $\lambda_{1}=\pi^{2}<11=-h^{\prime}(0)$ holds, cf. [3].

Our goal is to stabilize Equation (4.5) for the specified parameters at the origin by an MPC controller. Note that the main ideas remain completely the same as in [3]. Moreover, we point out that the following line of arguments is crucially based on the fact that optimality of the involved control law is not required in our controllability condition Assumption 2.2. In order to deduce Inequality (2.5) and, thus, exponential controllability in terms of the stage costs, a linear feedback control $u(x, t):=-K z(x, t)$ with a real constant $K$ is chosen. Then, Theorem 4.1 applied to the reaction diffusion equation given by (4.1), (4.2) with nonlinearity $h(z)+K z$ yields

$$
\ell^{\star}(z(\cdot, t))=\|z(\cdot, t)\|_{\mathcal{L}^{2}(\Omega)}^{2} \leq M^{2} e^{-2 \gamma t} \ell^{\star}\left(z_{0}(\cdot)\right)
$$

with $\gamma=\lambda_{1}+h^{\prime}(0)+K$. Furthermore, we get

$$
\begin{aligned}
\ell(z(\cdot, t), u(\cdot, t)) & =\|z(\cdot, t)\|_{\mathcal{L}^{2}(\Omega)}^{2}+\lambda\|u(\cdot, t)\|_{\mathcal{L}^{2}(\Omega)}^{2} \\
& =\|z(\cdot, t)\|_{\mathcal{L}^{2}(\Omega)}^{2}+\lambda K^{2}\|z(\cdot, t)\|_{\mathcal{L}^{2}(\Omega)}^{2}=\left(1+\lambda K^{2}\right) \ell^{\star}(z(\cdot, t)) .
\end{aligned}
$$

Combining this equation with (4.6) implies the desired exponential controllability

$$
\ell(z(\cdot, t), u(\cdot, t))=\left(1+\lambda K^{2}\right) \ell^{\star}(z(\cdot, t)) \leq C e^{-\mu t} \ell^{\star}\left(z_{0}(\cdot)\right)
$$

with overshoot $C:=\left(1+\lambda K^{2}\right) M^{2}$ and decay rate $\mu:=2 \gamma$. We point out the ambiguous role of the feedback gain $K$. Picking larger values for $K$ leads to faster decay but also to an increased overshoot - a trade-off. At this point, Formula (2.6) is used in order to formulate the following optimization problem.

Problem 4.2. Let $T>\delta>0, \lambda>0$, and $M \geq 1$ be given. Then,

$$
\underset{K \in \mathbb{R}}{\operatorname{maximize}} \alpha_{T, \delta} \text { given by }(2.6)
$$


with $C=\left(1+\lambda K^{2}\right) M^{2}=\left(1+0.01 \cdot K^{2}\right)$ and $\mu=2 \gamma=2\left(\pi^{2}-11+K\right)$ subject to

$$
\gamma=\lambda_{1}+h^{\prime}(0)+K=\pi^{2}-11+K \geq 0 .
$$

Here, a regularization parameter $\lambda=0.01$ and control horizon $\delta=0.01$ are used. Solving this nonlinear optimization problem shows that the minimal prediction horizon with guaranteed stability is $T \approx 0.0755$, cf. Figure 4.1 . Here, we restrict ourselves to prediction horizons $T$ which are multiple of the control horizon $\delta$. A prediction horizon of length $T=8 \delta$ guarantees a (strictly) positive suboptimality index $\alpha_{T, \delta}$ via (2.6). Note that the choice of $K$ only influences the resulting estimates on the overshoot $C$ and the decay rate $\sigma$ in Assumption 2.2, but does not appear in the MPC algorithm. In addition, since $\|z(\cdot, t)\|_{\mathcal{L}^{2}(\Omega)}^{2}=\ell^{\star}(z(\cdot, t))$ holds, Condition
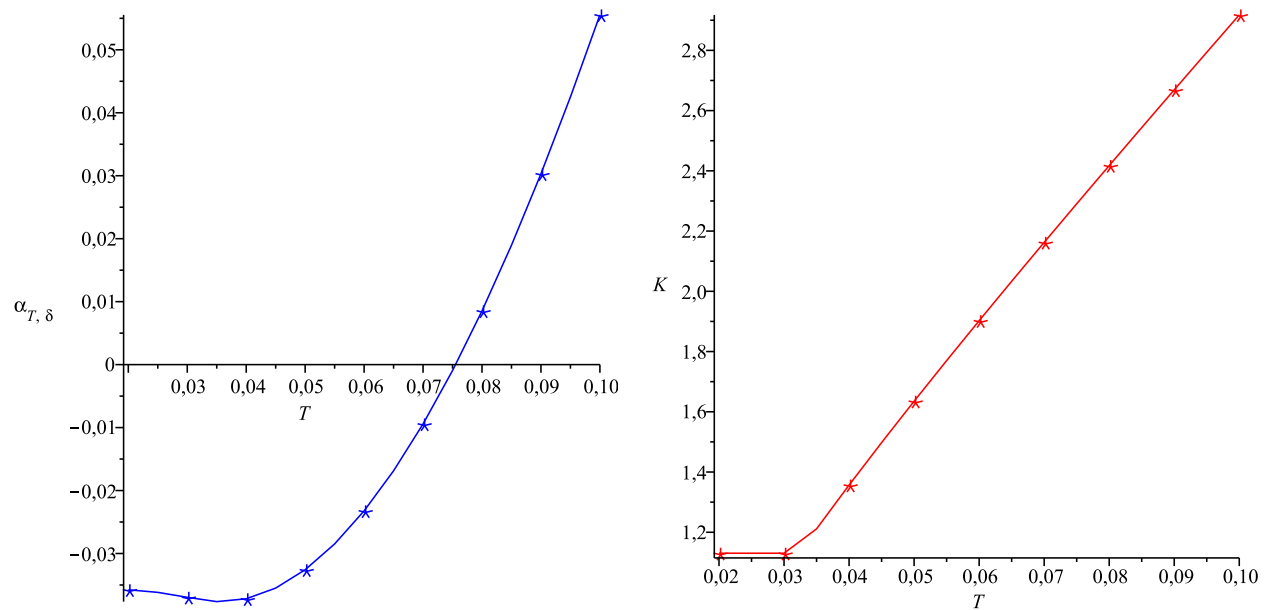

FIG. 4.1. On the left, the maximal achievable $\alpha_{T, \delta}$ for a given prediction horizon $T$ is depicted. According to this, the minimal stabilizing horizon, i.e., the smallest $T$ ensuring asymptotic stability, is obtained for $T \approx 0.0755$. On the right, we have illustrated the corresponding optimal choice of the parameter $K$ for given $T$.

(2.4) is satisfied for $\eta_{1}(r)=r^{2}$ and $\eta_{2}(r)=\int_{0}^{T} C e^{-\mu t} d t \cdot r^{2}$ in view of (2.5). Hence, asymptotic stability of the MPC controlled closed-loop can be ensured by applying Theorem 2.3 with prediction horizon $T=8 \delta$.

REMARK 4.3. Incorporating the controllability condition from [28] in our setting allows to choose the gain parameter $K$ for each parameter $\gamma_{i}, i \in \mathbb{N}_{0}$, independently. This results in better bounds in the linear program deduced in [9] and, thus, the corresponding Formula $\alpha_{N, m}$ presented in Theorem (2.6). As a consequence, tighter bounds on the required prediction horizon are obtained, cf. [29, Section 5.4] - an approach which can be directly transferred to the continuous time setting of Subsection 2.1.

Next, the continuous time system is discretized in order to apply Theorem 2.6. Here, the concept of multistep feedback laws of Definition 2.4 is crucial in order reproduce the continuous time system behavior for various discretization parameters $\tau$. Then, the connection of the corresponding estimates is investigated in order to illustrate our main results Theorem 3.2, Proposition 3.3, and Corollary 3.4.

Let $w(n)=\left.\tilde{u}(t)\right|_{[0, \tau)}$ be an element of $\mathcal{L}^{\infty}([0, \tau), \mathbb{U})$. Then, the solution of the finite horizon optimal control problems used in order to generate the MPC feedback 
is contained in $W(0, T)$. Since $W(0, T)$ is continuously embedded in $\mathcal{C}\left([0, T), \mathcal{L}^{2}(\Omega)\right)$ taking snapshots of the solution is well defined. This allows to choose the following stage costs

$$
\ell_{\tau}(z(n), w(n))=\tau\|z(n)\|_{\mathcal{L}^{2}(\Omega)}^{2}+\lambda \int_{0}^{\tau} \int_{\Omega} w(n)(x, t)^{2} d x d t
$$

which approximate the continuous time stage cost (4.3) on the sampling interval $[0, \tau)$, i.e.

$$
\ell_{\tau}(z(n), w(n)) \approx \int_{0}^{\tau} \| z_{w(n)}\left(t ; z(n) \|_{\mathcal{L}^{2}(\Omega)}^{2} d t+\lambda \int_{0}^{\tau} \int_{\Omega} w(n)(x, t)^{2} d x d t .\right.
$$

Repeating the line of arguments used in the continuous time setting yields $\ell_{\tau}^{\star}(z(n)) \leq$ $\sigma \ell_{\tau}^{\star}\left(z_{0}\right)$ with decay rate $\sigma=e^{-\mu \tau}$. Assuming $\lambda_{1}(\Omega)+h^{\prime}(0)+K \geq 0$ the following calculation shows that the same overshoot bound $C$ as for the continuous time setting holds:

$$
\begin{aligned}
\ell_{\tau}(z(n), w(n)) & =\tau\|z(n)\|_{\mathcal{L}^{2}(\Omega)}^{2}+\lambda \int_{0}^{\tau}\|\underbrace{w(n)(\cdot, t)}_{=u(\cdot, n T+t)=K z(\cdot, n T+t)}\|_{\mathcal{L}^{2}(\Omega)}^{2} d t \\
& <\tau\|z(n)\|_{\mathcal{L}^{2}(\Omega)}^{2}+\lambda \int_{0}^{\tau} K^{2} M^{2}\|z(\cdot, n T)\|_{\mathcal{L}^{2}(\Omega)}^{2} d t \\
& =\left(1+\lambda K^{2}\right) \tau\|z(n)\|_{\mathcal{L}^{2}(\Omega)}^{2}=C \ell_{\tau}^{\star}(z(n)) .
\end{aligned}
$$

Using the discretization parameter $\tau=0.01$, which corresponds to using $\delta$ as sampling time, Theorem 2.6 yields asymptotic stability for $N=10$. Hence, a prediction horizon of length $N \tau=0.1>0.0755$ is required. On the other hand, the discretization parameter $\tau=0.005=\delta / 2$ already improves this estimate and ensures asymptotic stability of the MPC closed-loop for $T=0.09$. Subdividing the interval $[0, \delta)$ into 8 parts, i.e., $\tau=\delta / 8$ further enhances the deduced bounds to $T=0.08$. We point out that this corresponds to the smallest multiple of $\delta$ for which asymptotic stability was guaranteed in the continuous time setting, cf. Figure 4.2. Summarizing, the suboptimality estimates computed for this example confirm our results: firstly, using a finer discretization and, thus, taking Assumption 2.5 more often into account yields improved performance bounds. Secondly, if a sufficiently fine discretization is employed the estimates deduced in the continuous time setting are reproduced.

4.2. Remarks on the Controllability Assumption. The most important ingredient in the stability analysis of unconstrained MPC is the controllability assumption, which is specified in Assumption 2.2 for the continuous time and in Assumption 2.5 for the discrete time case. Several examples were presented on how to verify this condition, e.g., in [10] for different linear and nonlinear ODE examples, and in [3] and Section 4.1 for PDE examples. In [8], two examples are shown for which the calculation of a feedback control law is difficult, but open-loop inputs are significantly simpler to obtain. Thus, the verification of the controllability assumption may be easier than the calculation of a control Lyapunov function as required for MPC with terminal weights.

With respect to the results presented in this work, it is particularly interesting how the two different controllability assumptions are connected. Theorem 3.2 suggests the following intuitive conjecture: If Assumption 2.2 is satisfied with decay rate $\mu>0$ and 

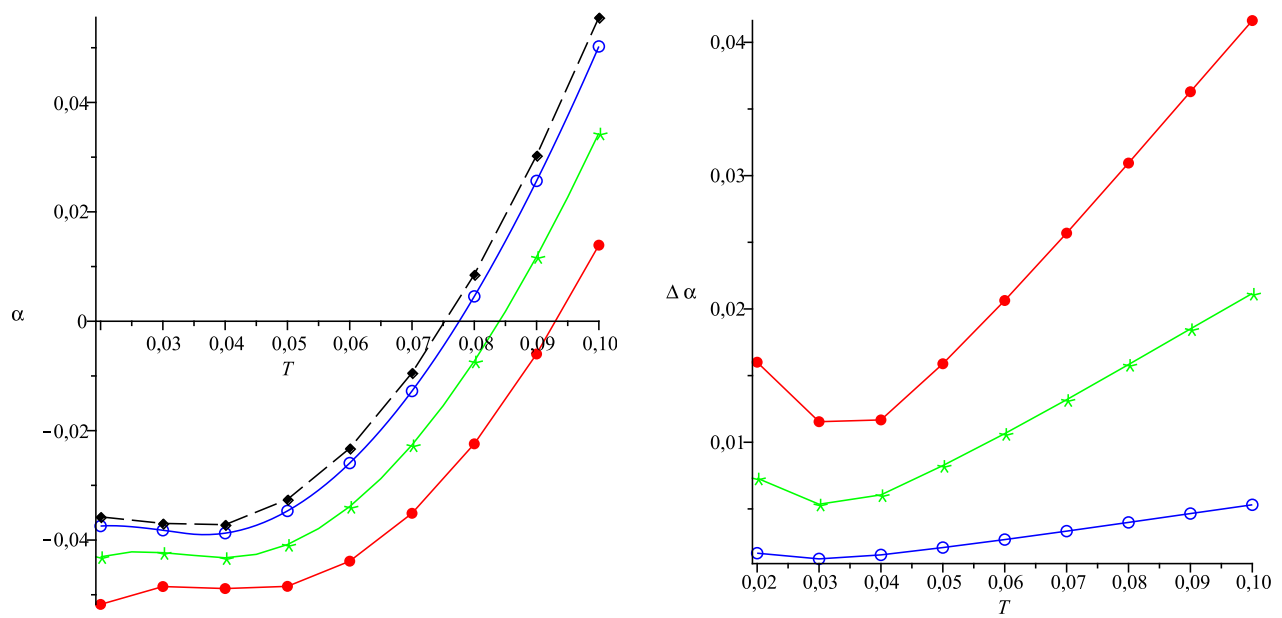

FIG. 4.2. On the left the suboptimality indices $\alpha$ resulting from Theorem 2.6 are depicted in red $(\tau=\delta, \bullet)$, green $(\tau=\delta / 2, \star)$, and blue $(\tau=\delta / 8, \circ)$. The dashed black line represents the performance estimates resulting from the continuous time setting. On the right, the deviation $\Delta \alpha$ from this continuous time reference trajectory is illustrated.

overshoot $C \geq 1$, then Assumption 2.5 holds with $\sigma:=e^{-\mu \tau}$ and the same overshoot $C$. For instance, consider quadratic stage costs similar to the one used in Section 4.1

$$
\begin{aligned}
& \ell(z(t), u(t))=\|z(t)\|^{2}+\lambda\|u(t)\|^{2}, \quad \quad \ell^{\star}\left(z_{0}\right)=\left\|z_{0}\right\|^{2}, \\
& \text { and } \quad \ell_{\tau}(z(n), w(n))=\tau\|z(n)\|^{2}+\lambda \int_{0}^{\tau}\|w(n)(t)\|^{2} d t, \quad \ell_{\tau}^{\star}\left(z_{0}\right)=\tau\left\|z_{0}\right\|^{2} .
\end{aligned}
$$

Using the control function from the continuous time Controllability Assumption 2.2 yields

$$
\begin{aligned}
\int_{n \tau}^{(n+1) \tau} \ell(z(t), u(t)) d t & =\int_{n \tau}^{(n+1) \tau}\left(\|z(t)\|^{2}+\lambda\|u(t)\|^{2}\right) d t \\
& \leq \int_{n \tau}^{(n+1) \tau} C e^{-\mu t}\left\|z_{0}\right\|^{2} d t<C e^{-\mu \tau n} \tau\left\|z_{0}\right\|^{2}=C \sigma^{n} \ell_{\tau}^{\star}\left(z_{0}\right) .
\end{aligned}
$$

Note that the integral was estimated by its maximum although the integral can easily be solved exactly. Since the state trajectory $z(\cdot)$ is continuous, $\int_{n \tau}^{(n+1) \tau}\|z(t)\|^{2} d t \approx$ $\tau\|z(n \tau)\|^{2}$ holds for sufficiently for small $\tau$. Using this approximation leads to the estimate

$$
\ell_{\tau}(z(n), w(n)) \leq(1+\mathcal{O}(\tau)) C \sigma^{n} \ell_{\tau}^{\star}\left(z_{0}\right) \quad \text { for } \sigma=e^{-\mu \tau} .
$$

Here $\mathcal{O}(\tau)$ denotes a quantity converging to zero for $\tau \rightarrow 0$. Hence, the discrete time Controllability Assumption 2.5 can be approximately concluded for sufficiently small sampling period $\tau$. An exact recovery of the controllability assumption is, in general, only possible in the limit of $\tau \rightarrow 0$. However, a significantly better characterization of the controllability properties is possible when considering a particular system. For instance, in Section 4.1 we showed for the Chafee-Infante equation that the discrete time controllability assumption can indeed be deduced from the continuous time counterpart with decay rate $\sigma=e^{-\mu \tau}$ and the same overshoot $C$. 
5. Conclusions. In $[9,10]$ a stability condition for unconstrained MPC was proposed. Although the approach introduced in [25, 24] extends this technique to a continuous time setting, a gap was observed between the corresponding results. In this paper we investigated the connection between both methodologies. We showed that the suboptimality estimates from [24] yield upper bounds on their discrete time counterparts. In addition, we proved that the estimates from $[9,10]$ are monotonically increasing for decreasing sampling period. Indeed, convergence to the bound obtained from the continuous time setting is rigorously shown. To this end, the concept of multistep feedback laws is essentiell.

Furthermore, we explained the practical relevance of the elaborated results. The continuous time result can be used in order to determine a theoretically achievable performance bound. Then, an iterative refinement can be employed in order to approximate this bound sufficiently well. In particular, a corresponding sampling period can be easily computed. This allows to specify a trade-off between sampling time and performance.

6. Appendix. In order to keep our paper sufficiently self-contained, we repeat here the result and proof of [29, Lemma 3.21] which is needed as an auxiliary result for the proof of Proposition 3.3.

Lemma 6.1. Let $m, M \in \mathbb{Z}$ with $M \geq m-1$ and constants $\gamma_{i} \in \mathbb{R}, i=m, m+$ $1, \ldots, M$ be given. Furthermore, the conventions $\prod_{i=m}^{m-1}=1$ and $\sum_{i=m}^{m-1}=0$ are used. Then, the following formula holds:

$$
\prod_{i=m}^{M} \gamma_{i}=\prod_{i=m}^{M}\left(\gamma_{i}-1\right)+\sum_{i=m}^{M}\left(\prod_{k=m}^{i-1} \gamma_{k} \prod_{k=i+1}^{M}\left(\gamma_{k}-1\right)\right) .
$$

Proof. We carry out an induction over $M$ in order to prove (6.1). Since we have agreed on the conventions with respect to the empty product and empty sum, the assertion holds for $M=m-1$. Hence, we proceed with the induction step:

$$
\begin{aligned}
\prod_{i=m}^{M+1}\left(\gamma_{i}-1\right) & =\left(\gamma_{M+1}-1\right) \prod_{i=m}^{M}\left(\gamma_{i}-1\right) \\
\stackrel{I . A .}{=} & \left(\gamma_{M+1}-1\right)\left[\prod_{i=m}^{M} \gamma_{i}-\sum_{i=m}^{M}\left(\prod_{k=m}^{i-1} \gamma_{k} \prod_{k=i+1}^{M}\left(\gamma_{k}-1\right)\right)\right] \\
& =\prod_{i=m}^{M+1} \gamma_{i}-\prod_{i=m}^{M} \gamma_{i}-\sum_{i=m}^{M}\left(\prod_{k=m}^{i-1} \gamma_{k} \prod_{k=i+1}^{M+1}\left(\gamma_{k}-1\right)\right) \\
& =\prod_{i=m}^{M+1} \gamma_{i}-\sum_{i=m}^{M+1}\left(\prod_{k=m}^{i-1} \gamma_{k} \prod_{k=i+1}^{M+1}\left(\gamma_{k}-1\right)\right)
\end{aligned}
$$

\section{REFERENCES}

[1] J. Ackermann. Sampled-Data Control Systems. Communications and Control Engineering Series. Springer-Verlag, Berlin, Heidelberg, 1985.

[2] F. Allgöwer and A. Zheng. Nonlinear model predictive control. Birkhäuser, Basel, 2000. 
[3] N. Altmüller, L. Grüne, and K. Worthmann. Receding horizon optimal control for the wave equation. In Proceedings of the 49th IEEE Conference on Decision and Control, pages 3427-3432, Atlanta, Georgia, USA, 2010.

[4] T. Cazenave and A. Haraux. An Introduction to Semilinear Evolution Equation. Oxford Science Publications, 1998.

[5] H. Chen and F. Allgöwer. A quasi-infinite horizon nonlinear model predictive control scheme with guaranteed stability. Automatica, 34(10):1205-1218, 1998.

[6] F. A. C. C. Fontes. A general framework to design stabilizing nonlinear model predictive controllers. Systems and Control Letter, 42(2):127-143, 2001.

[7] K. Graichen and A. Kugi. Stability and incremental improvement of suboptimal MPC without terminal constraints. IEEE Transactions on Automatic Control, 55(11):2576-2580, 2010.

[8] G. Grimm, M. J. Messina, S. E. Tuna, and A. R. Teel. Model predictive control: for want of a local control Lyapunov function, all is not lost. IEEE Transactions on Automatic Control, 50(5):546-558, 2005.

[9] L. Grüne. Analysis and design of unconstrained nonlinear MPC schemes for finite and infinite dimensional systems. SIAM J. Control Optim., 48(2):1206-1228, 2009.

[10] L. Grüne, J. Pannek, M. Seehafer, and K. Worthmann. Analysis of unconstrained nonlinear MPC schemes with time-varying control horizon. SIAM J. Control Optim., 48(8):49384962, 2010.

[11] L. Grüne and A. Rantzer. On the infinite horizon performance of receding horizon controllers. IEEE Transactions on Automatic Control, 53(9):2100-2111, 2008.

[12] B. Hu and A. Linnemann. Toward infinite-horizon optimality in nonlinear model predictive control. IEEE Transactions on Automatic Control, 47(4):679-682, 2002.

[13] A. Jadbabaie and J. Hauser. On the stability of receding horizon control with a general terminal cost. IEEE Transactions on Automatic Control, 50(5):674-678, 2005.

[14] S.S. Keerthi and E.G. Gilbert. Optimal infinite horizon feedback laws for a general class of constrained discrete-time systems: stability and moving horizon approximations. J. Optim. Theory Appl., 57:265-293, 1988.

[15] S. Lang. Real and Functional Analysis, volume 142 of Graduate Texts in Mathematics. Springer, New York, third edition, 1993.

[16] D. Limon, T. Alamo, F. Salas, and E. F. Camacho. On the stability of constrained MPC without terminal constraint. IEEE Transactions on Automatic Control, 51(5):832-836, 2006.

[17] B. Lincoln and A. Rantzer. Relaxing dynamic programming. IEEE Transactions on Automatic Control, 51(8):1249-1260, August 2006.

[18] D. Q. Mayne, J. B. Rawlings, C. V. Rao, and P. O. M. Scokaert. Constrained model predictive control: stability and optimality. Automatica, 26(6):789-814, 2000.

[19] P. Naittaanmäki and D. Tiba. Optimal Control Of Nonlinear Parabolic Systems. Marcel Dekker, Inc, 1994.

[20] D. Nešić and A.R. Teel. A framework for stabilization of nonlinear sampled-data systems based on their approximate discrete-time models. IEEE Transactions on Automatic Control, 49(7):1103-1122, 2004.

[21] S. J. Qin and T. A. Badgwell. A survey of industrial model predictive control technology. Control Engineering Practice, 11(7):733-764, 2003.

[22] T. Raff, S. Huber, Z. K. Nagy, and F. Allgöwer. Nonlinear model predictive control of a four tank system: An experimental stability study. In Proceedings of the IEEE Conference on Control Applications, pages 237-242, Munich, Germany, 2006.

[23] J. B. Rawlings and D. Q. Mayne. Model Predictive Control: Theory and Design. Nob Hill Publishing, Madison, WI, USA, 2009.

[24] M. Reble and F. Allgöwer. Unconstrained model predictive control and suboptimality estimates for nonlinear continuous-time systems. Accepted for publication in Automatica, 2011.

[25] M. Reble and F. Allgöwer. Unconstrained nonlinear model predictive control and suboptimality estimates for continuous-time systems. In Proceedings of the 18th IFAC World Congress, pages 6733-6738, Milan, Italy, 2011.

[26] M. Reble, M. A. Müller, and F. Allgöwer. Unconstrained model predictive control and suboptimality estimates for nonlinear time-delay systems. In Proceedings of the 50th IEEE Conference on Decision and Control and European Control, pages 7599-7604, Orlando, Florida, USA, 2011.

[27] F. Tröltzsch. Optimale Steuerung partieller Differentialgleichungen. Vieweg \& Sohn Verlag/GWV Fachverlage GmbH, Wiesbaden, 2005.

[28] S. E. Tuna, M. J. Messina, and A. R. Teel. Shorter horizons for model predictive control. In Proceedings of the American Control Conference, Minneapolis, Minnesota, USA, 2006. 
[29] K. Worthmann. Stability Analysis of unconstrained RHC. PhD thesis, University of Bayreuth, 2011. 